\title{
Observing Relative Motion With Three Accelerometer Triads
}

\author{
Patrick Schopp, Hagen Graf, Michael Maurer, Michailas Romanovas, \\ Lasse Klingbeil, and Yiannos Manoli, Senior Member, IEEE
}

\begin{abstract}
A gyroscope-free inertial measurement unit (GF-IMU) detects the relative motion of a body based on acceleration measurements only. It consists of multiple transducers attached at distinct locations within the body that together form an accelerometer array. In this paper, we employ only three accelerometer triads in order to completely capture the transversal and angular acceleration as well as the angular velocity. By modeling the GF-IMU as a nonlinear control system, we are able to conduct an observability analysis, which shows that this approach is capable of capturing an arbitrary spatial motion. We also show that additional triads only provide redundant information. Based on the control system formulation, we derive the models required to employ a nonlinear Kalman filter as a state observer. As the system description is of a general form they are suitable for any accelerometer array regardless of the number and placement of the transducers. Hence, the presented Kalman filter approach is applicable to all observable GF-IMU configurations. The measurements taken with a prototype on a 3-D rotation table confirm the observability analysis. The evaluations also show that the approach using three accelerometer triads achieves an estimation accuracy comparable with that of arrays employing a higher number of triads.
\end{abstract}

Index Terms-Accelerometer array, angular velocity, gyroscope-free inertial measurement unit (GF-IMU), nonlinear Kalman filter, observability.

\section{INTRODUCTION}

A $\mathrm{N}$ INERTIAL measurement unit (IMU) determines the relative motion of a body in the form of its transversal acceleration and its angular velocity. Such devices are widely used in applications that require the accurate knowledge of the position of a moving object, e.g., a ground vehicle [1] or an aircraft [2]. The relative motion measurement provided by

Manuscript received January 10, 2014; revised April 7, 2014; accepted May 13, 2014. Date of publication May 27, 2014; date of current version November 6, 2014. The Associate Editor coordinating the review process was Dr. Jesus Urena.

P. Schopp, H. Graf, and M. Maurer are with the Fritz Huettinger Chair of Microelectronics, Department of Microsystems Engineering, University of Freiburg, 79110 Freiburg, Germany (e-mail: patrick.schopp@imtek.unifreiburg.de).

M. Romanovas is with the German Aerospace Center, Institute of Communications and Navigation, 17235 Neustrelitz, Germany.

L. Klingbeil is with the Institute of Geodesy and Geoinformation, University of Bonn, 53115 Bonn, Germany.

Y. Manoli is with the Fritz Huettinger Chair of Microelectronics, Department of Microsystems Engineering, University of Freiburg, 79110 Freiburg, Germany, and also with the Institute of Micromachining and Information Technology, 78052 Villingen-Schwenningen, Germany.

Color versions of one or more of the figures in this paper are available online at http://ieeexplore.ieee.org.

Digital Object Identifier 10.1109/TIM.2014.2327472 an IMU is either used for pure inertial navigation or merged with the measurements of an absolute positioning system, such as the global positioning system [3], [4]. Nowadays, micromachined inertial sensors are available in very small packages at low cost. The reduced size and high availability revealed new consumer applications, as the devices can be integrated into everyday things, e.g., mobile phones or even clothes [5], [6]. Examples of those applications are pedestrian tracking [7], activity classification [8], or sports analysis [9].

A conventional IMU comprises three accelerometers and three gyroscopes. A gyroscope-free inertial measurement unit (GF-IMU) employs only accelerometers in order to detect the relative body motion. Multiple sensors are attached at fixed positions relative to each other within a rigid body. Therefore, a GF-IMU is also referred to as an accelerometer array.

The reasons for employing accelerometer arrays are manifold. In the following, we mention only two of the many interesting advantages of a GF-IMU over a conventional IMU. A micromachined acceleration sensor can be designed as a mechanically passive device [10], [11]. The deflection of a proof mass is measured to determine the occurring acceleration. Micromechanical gyroscopes exploit the Coriolis force that acts on a oscillating proof mass when an angular motion is present [12], [13]. Due to the required mechanical excitation, the power consumption of a gyroscope is $\sim 20$ times higher compared with an accelerometer. An accelerometer array can therefore be used to implement a system with a lower power consumption, even if a redundant number of transducers is used. This is particularly valuable for hand-held devices. Furthermore, the angular acceleration can be measured directly, thus avoiding the noiseamplification problem associated with the indirect measurement by differentiation [14]. In [15], this advantage was used to develop a hand-held microsurgical instrument which incorporates several accelerometers in order to detect the tremor of its user. Furthermore, accelerometer arrays have been successfully employed in applications, such as motion analysis during sports activities [16], [17] and impact situations [18], [19], or automotive navigation [20], [21], which confirm that accelerometer arrays are a viable alternative to a conventional IMU.

A fundamental question concerning the GF-IMU concept is the number of required transducers. The majority of publications concerned with accelerometer arrays state that at least 12 acceleration sensitive axes are necessary to directly determine 


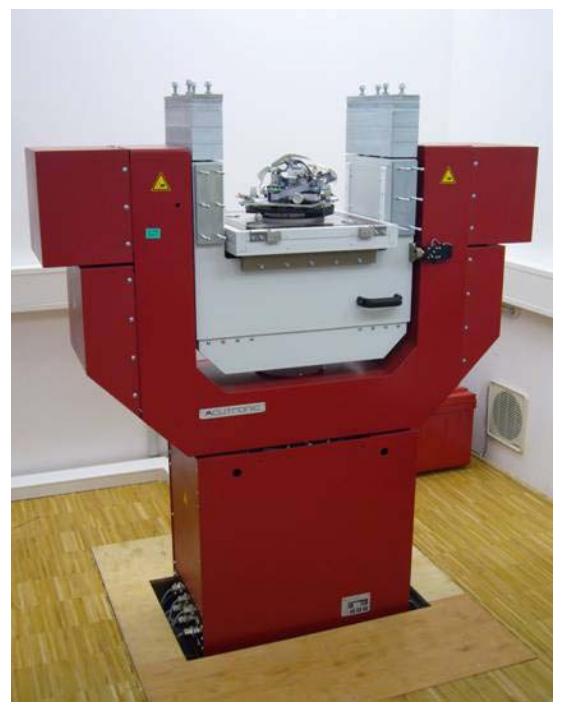

(a)

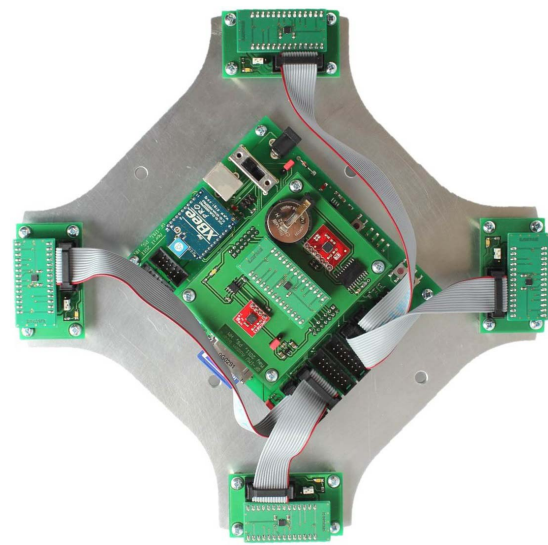

(b)

Fig. 1. Experimental setup. We use a (a) 3-D rotation table to impose a defined motion on the (b) designed GF-IMU prototype.

the angular velocity for a 3-D motion. This can be justified by an inverse kinematic formulation [22]. In this paper, we are concerned with an accelerometer array of only nine axes. By intuition, this is the minimum number of sensors, because the relative motion is described by nine state variables. However, previous attempts to determine the relative motion using a nine axes configuration could not provide a unique, singularity free solution. In our approach, we consider the motion dynamics as an integral part of the measurement system. Thus, we are able to show that relative motion is trackable with only nine transducers and provide a suitable observer. As three sensors can be saved this leads to a lower system cost and lower power consumption.

This paper is organized as follows. After the kinematic fundamentals are introduced in Section II, we discuss the existing approaches to a GF-IMU and state the novelty of this paper compared with the state of the art in Section III. Thereafter, we give a general system description of an arbitrary accelerometer array in Section IV before proving that an array of three sensor triads is generally capable to infer its relative motion in Sections V-VII. In Section VIII, we derive the

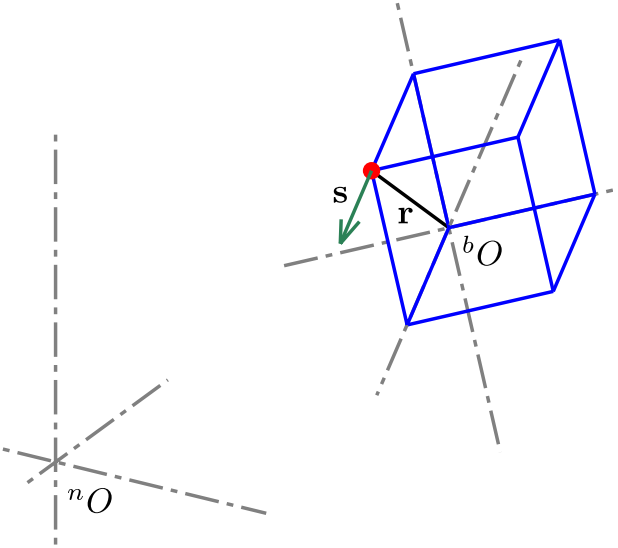

Fig. 2. Sensitive axis $\boldsymbol{s}$ of an accelerometer located at position $\boldsymbol{r}$ within a rigid body ${ }^{b} O$ relative to the navigation frame ${ }^{n} O$.

models required to employ a nonlinear Kalman filter as a state observer. Before we conclude this paper, we present explanatory experiments that validate the theory in Section IX. These were conducted on a 3 -D rotation table using a GF-IMU prototype we have designed (Fig. 1).

\section{KinEMATIC FUnDAMENTALS}

The acceleration field of a body is excited by its relative motion. Considering a rigid body undergoing a spatial motion (Fig. 2), the acceleration ${ }^{r} \boldsymbol{a}$ occurring at position $\boldsymbol{r}$ within the body frame ${ }^{b} \mathrm{O}$ can be calculated as

$$
{ }^{r} \boldsymbol{a}={ }^{b} \boldsymbol{a}+\dot{\boldsymbol{\omega}} \times \boldsymbol{r}+\boldsymbol{\omega} \times(\boldsymbol{\omega} \times \boldsymbol{r})
$$

where the relative body motion is defined in terms of the linear acceleration ${ }^{b} a$, the angular velocity $\boldsymbol{\omega}$, and the angular acceleration $\dot{\omega}$, all described in ${ }^{b} O$. Equation (1) is a sum of three distinct accelerations. Besides ${ }^{b} \boldsymbol{a}$, the tangential acceleration $\dot{\omega} \times \boldsymbol{r}$ and the centripetal acceleration $\boldsymbol{\omega} \times(\boldsymbol{\omega} \times \boldsymbol{r})$ are affecting ${ }^{r} \boldsymbol{a}$. The accelerations excited by the relative motion are different at distinct points $r$ in the body. The concept behind a GF-IMU exploits this fact. Several transducers distributed at multiple positions in ${ }^{b} O$ sample the acceleration field. Subsequently, the relationship described in (1) is employed to determine the relative motion of ${ }^{b} O$. The fundamental question that arises is how many accelerometers are necessary and how they must be placed in order to capture the relative motion.

\section{RELATED WORK}

The common way to derive the relative motion by means of the acceleration field is the inverse kinematic approach. For each sensor, (1) is applied to construct a system of equations. This system is solved for the variables of the relative motion. The result is a computation rule by which the acceleration values can be processed. The published approaches can be separated into two groups: solutions that determine the angular velocity indirectly or directly. 
The indirect methods only gather the linear and angular accelerations directly. The angular velocity must be calculated by integration of the angular acceleration. The minimum number of single axes sensors for this approach is six [23]-[25]. However, the inverse kinematic solution for the angular acceleration involves prior knowledge of the angular velocity. As the angular velocity is prone to integration errors, the computation is potentially unstable. To address this issue, methods were proposed to eliminate the angular velocity terms from the equations to calculate the angular acceleration. For example, this can be achieved by means of an additional triaxial sensor [24] or by arranging the transducers in a special cube configuration [26], [27]. Nonetheless, the angular velocity can only be determined with an unbounded error due to the mandatory integration step of the angular acceleration.

The direct methods attempt to estimate the angular acceleration and velocity simultaneously. This allows for a drift-free estimation of the angular velocity. However, additional sensors are necessary. Furthermore, the centripetal force in (1) depends quadratically on the angular velocity. Thus, the acceleration field is independent of the sign, i.e., the direction of the rotation. As a consequence, an inverse kinematic approach that computes an estimate of the angular velocity based on one set of acceleration values cannot determine the direction of the present rotation. Specifically, an equation system constructed by (1) can only be solved uniquely for six quadratic terms of the angular velocity, which are permutations of the three major rotation axes. To recover the sign of the rotation, additional techniques must be added that merge the obtained angular acceleration and quadratic terms. There is no clear consensus about the minimal number of acceleration sensitive axes in literature. Approaches were published proposing either nine or 12 accelerometers.

Genin et al. [28] investigated an array of single axes sensors configured as three triplets of parallel axes. Applying an inverse kinematic approach he concludes that nine transducers are sufficient to obtain the quadratic terms of the angular velocity. However, the solution he obtains is not unique. Extending the work of Genin, closed-form expressions for an arbitrary placement of the nine single axes sensors have been developed in [29] and [30]. Given a set of accelerations and provided that the sensor setup is feasible, these result in eight possible solutions for the angular motion. In addition, the derived closed-form expressions are difficult to expand to higher, more redundant number of accelerometers. Park et al. [31], [32] introduced another interesting methodology to estimate the angular velocity using nine sensors. First, they obtain the linear and angular acceleration from a six sensor configuration and perform an integration step to gather the angular velocity. To deal with the unbounded drift error, they add a separate triaxial accelerometer, which they refer to as observer-accelerometer. The measurements of this device are continuously predicted based on the current estimate of the relative motion. They form a closed loop system by correcting the angular velocity according to the difference between predicted and actual observation. In [31], the cube configuration is used for the six sensor setup, whereas in [32] they propose a coplanar setup. However, although they present promising simulations, they cannot prove that their method is capable to estimate the relative movement with a bounded error in every possible situation.

Zappa et al. [22] commented on the work of Genin and raised the following issues. In accordance with Genin, [22] identifies two possible ways to compute an inverse kinematic solution for a configuration of nine sensors. He then argues that the first one is singular when one or more quadratic terms of the angular velocity are zero and that the solution of the second one is not unique as eight are always found. $\mathrm{He}$ concludes that for a unique and singularity free solution at least 12 accelerometers must be used. In addition to his inverse kinematic approach, he states geometric rules for the placement of the transducers. Those rules forbid a coplanar setup of the sensors.

All proposed sensor configurations to a 12 axes GF-IMU known to us comply with the rules of Zappa. They differ in the computation of the relative motion and in particular in the way the sign of the rotation is recovered. The simplest approach to determine the direction of the rotation is the integration of the gathered angular acceleration [33]. Of course, drift errors are inevitable. To avoid these, the quadratic terms of the angular velocity can be used to obtain the magnitude of the rotation while only the direction is determined involving the integrated angular acceleration [34]-[37]. In contrast to this, recursive Bayesian filters, like the Kalman filter, allow to recover the magnitude and direction of the rotation jointly. Thereby, the uncertainty of the used models and the acceleration measurements are considered within the filtering process, which results in an efficient noise cancelation and a robust estimation behavior. In our previous work, we employ an unscented Kalman filter (UKF) to merge the measurements of all 12 sensors [21], [38], [39]. Nonetheless, other nonlinear variants of the Kalman filter are also applicable. For example, Edwan uses an extended Kalman filter (EKF) to determine the rotation in [40]. In a similar fashion, Park processes the sensor data with an EKF in [41]. There, he also comments on his previous work [31]. He states that his approach using only nine sensors has a deficient estimation behavior if the angular velocity is zero in one dimension. Therefore, he employs an accelerometer array with twelve sensitive axes that complies to the rules of Zappa.

In summary, there is no clear consensus about the minimal number of transducers necessary to directly estimate the angular velocity. Although promising approaches using only nine accelerometers were published, they all suffer from certain shortcomings. Either the solution resulting from the inverse kinematic approach is not unique or a flawless estimation cannot be guaranteed. Until now, this was only achieved for arrays consisting of 12 sensors. With this paper, we contribute to this topic by the following. First, we model the accelerometer array as a control system. Its internal states are the relative body motion, its outputs are the acceleration measurements. The number of transducers and their placement remain arbitrary in this description. The benefit of this is that the system dynamics are incorporated intrinsically. Then, we use this system formulation to model an accelerometer 
array of nine sensors in a reasonable geometric configuration. For this nonlinear system, we perform an analytical investigation on the observability of the system state. Concretely, we show that the system has the property of local weak observability (LWO). Thus, it is possible to track the relative body motion, including the sign of the angular velocity.

This result leads to the following advancements. A GF-IMU is feasible with only nine accelerometers. Thus, three sensors can be saved. In contrast to the rules derived in [22], the transducers can be mounted on a surface, which facilitates the integration for many applications, e.g., hand-held devices. As LWO for the given system is shown any valid observer can be employed. Here, we show how to apply a nonlinear Kalman filter. The integration of the models is straightforward and the derived models can be used for any GF-IMU configuration as the original control system formulation holds true for an arbitrary sensor array. Furthermore, the derived models are suitable for any nonlinear Kalman filter variant. As there is no need to derive an inverse kinematic solution, the incorporation of additional sensors becomes trivial. In addition, we show that the observability analysis for a configuration of 12 transducers leads to the same results as for a configuration of nine sensors. Nonetheless, additional sensors may increase the estimation accuracy, which we demonstrate by our experiments.

\section{SySTEM DESCRIPTION}

The behavior of many technical systems can be modeled by the following continuous-time state-space formulation [42]:

$$
\Sigma: \begin{aligned}
& \dot{x}=f(x, u) \\
& y=h(x)
\end{aligned}
$$

where $\boldsymbol{x} \in \mathbb{R}^{n}$ is the state of system $\Sigma, \boldsymbol{y} \in \mathbb{R}^{m}$ is the observation, and $\boldsymbol{u} \in \mathbb{R}^{l}$ is the control input. Both the process model $\boldsymbol{f}$ and the observation model $\boldsymbol{h}$ are infinitely differentiable vector functions. In the following, we use this formulation to describe the GF-IMU as a control system. Thus, we define the state and derive the models for an arbitrary sensor array.

The observation model $\boldsymbol{h}$ provides a measurement $\boldsymbol{y}$ for any state $\boldsymbol{x}$. Since the concept of a GF-IMU is to determine the relative body motion using only accelerometers, the sensor equations of these transducers form the basis of the observation model. The acceleration ${ }^{s} a_{i}$ that is measured by a singleaxis sensor $S_{i}$ fixed at position $\boldsymbol{r}_{i}$ can be calculated by

$$
\begin{aligned}
{ }^{s} a_{i} & =\boldsymbol{s}_{i} \cdot{ }^{r} \boldsymbol{a}_{i}+o_{i} \\
& =\boldsymbol{s}_{i}^{T}\left({ }^{b} \boldsymbol{a}+\dot{\boldsymbol{\omega}} \times \boldsymbol{r}_{i}+\boldsymbol{\omega} \times\left(\boldsymbol{\omega} \times \boldsymbol{r}_{i}\right)\right)+o_{i}
\end{aligned}
$$

where $o_{i}$ is the signal offset of the respective transducer. Vector $s_{i}$ is the sensitivity axis of the accelerometer described in the body frame ${ }^{b} O$. Thus, it encodes both the sensitivity of the sensor by its length as well as its orientation by its direction. To generate the scalar acceleration measurement ${ }^{s} a_{i}$, the 3-D acceleration ${ }^{r} \boldsymbol{a}_{i}$ occurring at position $\boldsymbol{r}_{i}$ is related with $s_{i}$ by their scalar product. Note that sensor model (3) can equally be used to model accelerometers that incorporate more than one sensitive axis in the same sensor housing by using several equations to calculate the sensor outputs. In the following, the term accelerometer triad will be used to address a triple-axis accelerometer.

The state of the system must fully cover all variables that influence the observations $\boldsymbol{y}$, as it is the only input to the observation model $\boldsymbol{h}$. For the GF-IMU, the system state $\boldsymbol{x}$ is thus defined as

$$
\boldsymbol{x}=\left[\begin{array}{lll}
{ }^{b} \boldsymbol{a}^{T} & \boldsymbol{\omega}^{T} & \dot{\boldsymbol{\omega}}^{T}
\end{array}\right]^{T}
$$

since the acceleration measurements are driven only by the variables of the relative body motion. Clearly, $\boldsymbol{x}$ cannot be observed by only one acceleration measurement. Hence, observation model $\boldsymbol{h}$ for the GF-IMU is constructed by applying $m$ sensor equations (3)

$$
\boldsymbol{y}=\boldsymbol{h}(\boldsymbol{x})=\left[\begin{array}{lll}
{ }^{s} a_{1} & \ldots & { }^{s} a_{m}
\end{array}\right]^{T} .
$$

The process model $f$ describes the behavior of the system and the interaction of the state variables. The derivative of the state $\dot{\boldsymbol{x}}$ is driven by the state of the system $\boldsymbol{x}$ itself and an external input $\boldsymbol{u}$. For the GF-IMU, the process model can be expressed as

$$
\dot{x}=f(x, u)=f_{1}(x)+f_{2}(u)=\left[\begin{array}{c}
0 \\
\dot{\omega} \\
0
\end{array}\right]+\left[\begin{array}{c}
u_{a} \\
0 \\
u_{\dot{\omega}}
\end{array}\right]
$$

where $f_{1}$ describes the impact of the current relative motion. Concretely, $f_{1}$ states that the angular acceleration is the time derivative of the angular velocity. The derivatives of the accelerations ${ }^{b} a$ and $\dot{\omega}$ are both zero in $f_{1}$ as they do not depend on the state $\boldsymbol{x}$. Instead, they are driven by the external inputs $\boldsymbol{u}_{a}$ and $\boldsymbol{u}_{\dot{\omega}}$, as expressed by $\boldsymbol{f}_{2}$. Although $\boldsymbol{u}_{a}$ and $\boldsymbol{u}_{\dot{\omega}}$ are unknown for most applications of a GF-IMU, it is still advantageous to introduce them to the process model as they later serve to consistently describe the dynamics of the motion within the state estimator.

The derived system model consisting of (4)-(6) is applicable to any accelerometer array. The specific approaches to a GF-IMU distinguish themselves in the number of sensors and the sensor placement. Thus, for every different setup, the observation model $\boldsymbol{h}$ has to include the respective number of sensor equations. The corresponding sensor positions $\boldsymbol{r}_{i}$ and sensitivity axes $\boldsymbol{s}_{i}$ have to be adjusted according to the placement of the sensors. However, not all possible accelerometer configurations are suitable to estimate the relative motion. Although a valid system description can be created, it is not possible to observe $\boldsymbol{x}$. A trivial example is an array of only one transducer. To ensure a proper estimation of the relative motion, the system must be analyzed whether its state is observable by the measurements.

\section{OBSERVABILITY}

A system is said to be observable if its initial state can be uniquely determined from a finite number of observations of its outputs and the knowledge of its inputs [43]. This also implies that the complete trajectory of the state through the state space can be reconstructed. For linear time-invariant 
systems, observability is always a global property. Thus, if the system is observable, every initial state can be distinguished from any other state. However, the observation model (5) of the GF-IMU system is nonlinear. For nonlinear systems, observability is not necessarily a global property. Here, the initial state may not be distinguishable from any other state, but only from states within its neighborhood. In that case, observability is a local property of the system. Still, this may be sufficient to track the state of the system with a suitable observer. In order to define a condition that indicates whether this is possible, Hermann and Krener introduced the concept of LWO in [44], which states the following. A certain point in state space is called LWO if this state is directly distinguishable from its neighbors. If LWO can be verified for all states, then the system is said to be locally weakly observable. The approach of Hermann and Krener was applied to various complex control systems. Examples can be found in robotics [45], state estimation of actuators [46], motion estimation [47], or calibration tasks [48]. For a detailed discussion on nonlinear observability we refer the reader to [49], [50].

Hermann and Krener also provide an algebraic test to check whether a system is LWO at a certain point, called the observability rank criterion. A few mathematical tools are necessary to apply the test, which we will introduce briefly. The observability rank criterion is based on the observation space $\mathcal{O}$, which is spanned by the output functions $y_{i}=h_{i}(\boldsymbol{x})$ and their time derivatives $\mathrm{d}^{k} y_{i} / \mathrm{d} t^{k}$ with $k$ being the order of the derivative. As the observation model $\boldsymbol{h}(\boldsymbol{x})$ is not dependent on time $t$, it is not differentiable with respect to $t$. However, the time derivative of state $\boldsymbol{x}$, which is the only input to $\boldsymbol{h}$, is described by the process model $\boldsymbol{f}$. Therefore, $\boldsymbol{f}$ is employed to formulate the time derivatives $\mathrm{d}^{k} y_{i} / \mathrm{d} t^{k}$ as Liederivatives $\mathcal{L}_{f}^{k} h_{i}$, which can be interpreted as the derivative of $h_{i}$ along the vector field of $\boldsymbol{f}$. Like this, the time derivatives of the observations can be described analytically and evaluated for a certain $\boldsymbol{x}_{0}$. Thus, we have $\mathrm{d}^{k} y_{i} /\left.\mathrm{d} t^{k}\right|_{\boldsymbol{x}}=\mathcal{L}_{f}^{k} h_{i}(\boldsymbol{x})$, which yields for the definition of the observation space

$$
\mathcal{O}=\operatorname{span}\left\{\mathcal{L}_{f}^{k} h_{i}(\boldsymbol{x}) \mid k \geq 0, \quad i=1, \ldots, m\right\} .
$$

Lie-derivatives can be defined recursively. Thereby, the zeroth-order Lie-derivative of the function $h_{i}$ with respect to $f$ is the function itself

$$
\mathcal{L}_{f}^{0} h_{i}(\boldsymbol{x})=h_{i}(\boldsymbol{x}) .
$$

The higher order Lie-derivatives are expressed by the scalar product

$$
\mathcal{L}_{f}^{k} h_{i}(\boldsymbol{x})=\left(\nabla \mathcal{L}_{f}^{k-1} h_{i}(\boldsymbol{x})\right) \cdot \boldsymbol{f}(\boldsymbol{x}) \quad k>0
$$

where the evaluation of the gradient operator $\nabla=\left[\partial / \partial x_{1} \ldots \partial / \partial x_{m}\right]^{T}$ generates a vector of all partial differentials. Hence, the Lie-derivative of a scalar function is again a scalar function.

Equation (7) states that the observation space $\mathcal{O}$ contains all linear combinations of the output functions and their time derivatives. As the state is of dimension $n$, there must exist at least $n$ linearly independent functions in $\mathcal{O}$ in order to instantaneously distinguish a certain point $\boldsymbol{x}_{0}$ from all its neighboring points. However, this condition is difficult to verify as there are infinitely many combinations in $\mathcal{O}$. Hermann and Krener [44] showed that a system is locally weakly observable at a certain point $\boldsymbol{x}_{0}$ if the dimension of the codistribution of $\mathcal{O}$ equals the dimension of the state. The codistribution $\nabla \mathcal{O}$ is spanned by the derivatives of the elements of $\mathcal{O}$ with respect to the elements of $\boldsymbol{x}$ and is thus defined as

$$
\nabla \mathcal{O}(\boldsymbol{x})=\operatorname{span}\left\{\nabla \mathcal{L}_{\boldsymbol{f}}^{k} h_{i}(\boldsymbol{x}) \mid k \geq 0, \quad i=1, \ldots, m\right\}
$$

which can be evaluated for any point $\boldsymbol{x}_{0}$ and is of matrix-form with $n$ columns an infinitely many rows. Thus, the observability rank condition results in the calculation of the rank of a matrix with infinitely many rows. However, Anguelova [51] showed that it is sufficient to include only the first $n-1$ Lie-derivatives for the rank computation. This leads to the following matrix $O_{M}(\boldsymbol{x})$, which is often referred to as the observability matrix:

$$
O_{M}(\boldsymbol{x})=\left[\begin{array}{l}
\nabla \mathcal{L}_{f}^{0} h_{1}(\boldsymbol{x}) \\
\vdots \\
\nabla \mathcal{L}_{f}^{0} h_{m}(\boldsymbol{x}) \\
\vdots \\
\nabla \mathcal{L}_{\boldsymbol{f}}^{n-1} h_{1}(\boldsymbol{x}) \\
\vdots \\
\nabla \mathcal{L}_{f}^{n-1} h_{m}(\boldsymbol{x})
\end{array}\right] .
$$

Furthermore, if at an instance $k$ the gradient $\nabla \mathcal{L}_{f}^{k} h_{i}(\boldsymbol{x})$ shows linear dependency on its corresponding lower order gradients, then this $k$ th instance as well as any further order gradients do not need to be included in the rank calculation.

However, the observability rank criterion is only a sufficient test for LWO. At certain singular points $O_{M}$ may not be full rank although the system is LWO. An intuitive example can be found in [49, Example 3.7].

\section{GEOMETRY}

Each measurement equation $h_{i}$ incorporates the parameters of its corresponding sensor, which are its position $\boldsymbol{r}_{i}$ and its sensitivity axis $s_{i}$. This yields to a sum of six free parameters for each single axis transducer. As we are concerned with a configuration of $m=9$ sensors, the observation model $\boldsymbol{h}$ of the entire accelerometer array incorporates in total 54 free parameters, that define the geometry of the array. In order to construct the observability matrix $O_{M}$ according to (11), the Lie-derivatives of $\boldsymbol{h}$ along $\boldsymbol{f}$ must be calculated. This introduces the geometry parameters of the array to $O_{M}$. Hence, the rank of $O_{M}$ strongly depends on these parameters. However, many of the possible combinations of these parameters clearly result in a nonobservable system. For example, if all sensors reside in one point or if all sensors are facing in the same direction. Therefore, we restrict the analysis to a certain placement of the sensors for the construction of the observability matrix. This simplifies the rank computation of $O_{M}$ since there is no need to consider the nonobservable 


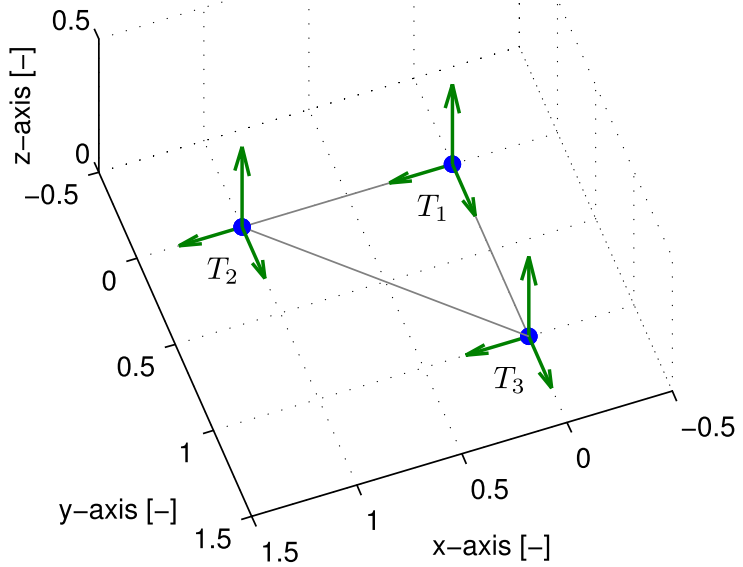

Fig. 3. Sensor configuration used for the observability analysis. The sensors triads $T_{1}, T_{2}$, and $T_{3}$ all reside on a surface, indicated by the triangle.

TABLE I

Positions AND SENSITIVITY AXES OF THE SENSORS USED FOR THE CONSTRUCTION OF $O_{M}$

\begin{tabular}{llll}
\hline Triad & Sensor & Position & \multicolumn{2}{l}{ Sensitivity axis } \\
$T_{j}$ & $S_{i}$ & $\boldsymbol{r}_{i}$ & $\boldsymbol{s}_{i}$ \\
\hline \multirow{4}{*}{$T_{1}$} & $S_{1}$ & & {$\left[\begin{array}{lll}1 & 0 & 0\end{array}\right]^{T}$} \\
& $S_{2}$ & {$\left[\begin{array}{lll}0 & 0 & 0\end{array}\right]^{T}$} & {$\left[\begin{array}{lll}0 & 1 & 0\end{array}\right]^{T}$} \\
& $S_{3}$ & & {$\left[\begin{array}{lll}0 & 0 & 1\end{array}\right]^{T}$} \\
\hline \multirow{3}{*}{$T_{2}$} & $S_{4}$ & & {$\left[\begin{array}{lll}1 & 0 & 0\end{array}\right]^{T}$} \\
& $S_{5}$ & {$\left[\begin{array}{lll}1 & 0 & 0\end{array}\right]^{T}$} & {$\left[\begin{array}{lll}0 & 1 & 0\end{array}\right]^{T}$} \\
& $S_{6}$ & & {$\left[\begin{array}{lll}0 & 0 & 1\end{array}\right]^{T}$} \\
\hline \multirow{4}{*}{$T_{3}$} & $S_{7}$ & & {$\left[\begin{array}{lll}1 & 0 & 0\end{array}\right]^{T}$} \\
& $S_{8}$ & {$\left[\begin{array}{llll}0 & 1 & 0\end{array}\right]^{T}$} & {$\left[\begin{array}{lll}0 & 1 & 0\end{array}\right]^{T}$} \\
& $S_{9}$ & & {$\left[\begin{array}{lll}0 & 0\end{array}\right]^{T}$} \\
\hline
\end{tabular}

parameter configurations. The restrictions to the geometry are related to a real life setup and represent a general meaningful configuration of the sensors. Still, they are universal enough to obtain a general understanding of the systems observability. The resulting sensor setup is shown in Fig. 3. In the following, we summarize the constraints we impose on the setup and state their background.

1) Three single axis sensors are grouped into one sensor triad $T_{j}, j=\{1,2,3\}$. Within one triad the sensors are fixed at the same position and their sensitive axes form an orthonormal basis. This constraint accounts for the fact that modern micromachined accelerometers usually incorporate three sensitive axes.

2) Sensor triad $T_{1}$ is fixed at the center of the body. This simplification can be applied without any restrictions to a viable sensor setup, as the center of the body frame can be chosen arbitrarily by applying a coordinate transformation.

3) The three positions of the triads span a plane. If this was not the case they would reside in one point or on a line. This would lead to a nonobservable configuration. To further simplify the computation of $O_{M}$ we set the positions of $T_{2}$ and $T_{3}$ at to a normalized unit length on the $x$-axis and $y$-axis.

4) All three triads are oriented such that their sensitive axes are parallel to the three major axes of the body. As the sensitive axes of one triad forms an orthonormal basis, the transducers observe a 3-D point acceleration.

A summary of the geometry parameters can be found in Table I. Applying the geometry parameters to (5), the GF-IMU observation model $\boldsymbol{h}(\boldsymbol{x})$ simplifies to

$$
\boldsymbol{h}(\boldsymbol{x})=\left[\begin{array}{l}
h_{1}(\boldsymbol{x}) \\
h_{2}(\boldsymbol{x}) \\
h_{3}(\boldsymbol{x}) \\
h_{4}(\boldsymbol{x}) \\
h_{5}(\boldsymbol{x}) \\
h_{6}(\boldsymbol{x}) \\
h_{7}(\boldsymbol{x}) \\
h_{8}(\boldsymbol{x}) \\
h_{9}(\boldsymbol{x})
\end{array}\right]=\left[\begin{array}{l}
{ }^{b} a_{x} \\
{ }^{b} a_{y} \\
{ }^{b} a_{z} \\
{ }^{b} a_{x}-\omega_{y}^{2}-\omega_{z}^{2} \\
{ }^{b} a_{y}+\omega_{x} \omega_{y}+\dot{\omega}_{z} \\
{ }^{b} a_{z}+\omega_{x} \omega_{z}+\dot{\omega}_{y} \\
{ }^{b} a_{x}+\omega_{x} \omega_{y}+\dot{\omega}_{z} \\
{ }^{b} a_{y}-\omega_{x}^{2}-\omega_{z}^{2} \\
{ }^{b} a_{z}+\omega_{y} \omega_{z}+\dot{\omega}_{x}
\end{array}\right]
$$

where we furthermore assume that the signal offset of all sensors equals zero.

\section{RANK TEST}

As described in Section V, we construct $O_{M}(\boldsymbol{x})$ by calculating the Lie-derivatives $\mathcal{L}_{f}^{k} h_{i}(\boldsymbol{x})$ until we find a linear dependency between the current order and their corresponding lower ones. The process model given in (6) describes the GF-IMU as a control system with known inputs $\boldsymbol{u}$. However, during the normal operation of the accelerometer array the motion that is imposed on the array is not controlled, i.e., the inputs $\boldsymbol{u}_{a}$ and $\boldsymbol{u}_{\dot{\omega}}$ remain unknown. This must be considered within the observability analysis, as known inputs are assumed here. Thus, instead of constructing the Lie-derivatives along the complete process model $f$, we only employ $f_{1}$, which describes the autonomous system behavior. The resulting matrix can be partitioned $O_{M}$ as

$$
O_{M}(\boldsymbol{x})=\left[\begin{array}{l}
\nabla \mathcal{L}_{f_{1}}^{0} h_{1}(\boldsymbol{x}) \\
\vdots \\
\nabla \mathcal{L}_{f_{1}}^{0} h_{9}(\boldsymbol{x}) \\
\hline \nabla \mathcal{L}_{f_{1}}^{1} h_{4}(\boldsymbol{x}) \\
\vdots \\
\nabla \mathcal{L}_{f_{1}}^{1} h_{9}(\boldsymbol{x}) \\
\hline \nabla \mathcal{L}_{f_{1}}^{2} h_{4}(\boldsymbol{x}) \\
\vdots \\
\nabla \mathcal{L}_{f_{1}}^{2} h_{9}(\boldsymbol{x})
\end{array}\right]=\left[\begin{array}{c|c|c}
I_{3 \times 3} & 0_{3 \times 3} & 0_{3 \times 3} \\
I_{3 \times 3} & A_{6 \times 3} & B_{6 \times 3} \\
I_{3 \times 3} & & \\
\hline 0_{3 \times 3} & \dot{A}_{6 \times 3} & A_{6 \times 3} \\
\hline 0_{3 \times 3} & & \\
\hline 0_{3 \times 3} & 0_{6 \times 3} & 2 \dot{A}_{6 \times 3} \\
0_{3 \times 3} & & \\
\end{array}\right]
$$

where the indexes $m \times n$ of the submatrices indicate their size with $m$ being the number of rows and $n$ the number of columns. $I_{m \times n}$ is the identity matrix and $0_{m \times n}$ a matrix with only zero entries. As Lie-derivatives of an order higher than $k=2$ are zero vectors there is 
no need to consider them. Submatrix $A_{6 \times 3}$ of $O_{M}(\boldsymbol{x})$ evaluates to

$$
A_{6 \times 3}=\left[\begin{array}{ccc}
0 & -2 \omega_{y} & -2 \omega_{z} \\
\omega_{y} & \omega_{x} & 0 \\
\omega_{z} & 0 & \omega_{x} \\
\omega_{y} & \omega_{x} & 0 \\
-2 \omega_{x} & 0 & -2 \omega_{z} \\
0 & \omega_{z} & \omega_{y}
\end{array}\right] .
$$

Its time derivative $\dot{A}_{6 \times 3}$ contains all entries of $A_{6 \times 3}$ but each entry is differentiated with respect to time $t$. Thus, $\dot{A}_{6 \times 3}$ is only dependent on the angular acceleration $\dot{\boldsymbol{\omega}}$. Submatrix $B_{6 \times 3}$ contains only constants and is given by

$$
B_{6 \times 3}=\left[\begin{array}{rrr}
0 & 0 & 0 \\
0 & 0 & 1 \\
0 & -1 & 0 \\
0 & 0 & -1 \\
0 & 0 & 0 \\
1 & 0 & 0
\end{array}\right] .
$$

In order to prove that $O_{M}$ is of full rank we show that every column is linearly independent from all other columns. For the sake of clarity, the argumentation can be found in Appendix. We summarize the rank determination of $O_{M}$ with the following statement. The observability matrix $O_{M}(\boldsymbol{x})$ is of rank $n=9$ for all $\boldsymbol{x}$, except for the case when $\boldsymbol{\omega}=\dot{\boldsymbol{\omega}}=\mathbf{0}$. Based upon the observability rank condition, the GF-IMU system is locally weakly observable for all motions except when, at the same time, no angular velocity and no angular acceleration are present. However, the point where $\boldsymbol{\omega}=\dot{\boldsymbol{\omega}}=\mathbf{0}$ is identifiable. It can be seen from (5) or (12) that at this point the accelerometers solely measure the transversal body acceleration ${ }^{b} \boldsymbol{a}$. Thus, all sensors are recording the same acceleration, since only $\omega$ and $\dot{\omega}$ are causing diverse accelerations within the body. As such, this point can be uniquely detected. It can therefore be considered as a singular point of the system where the rank condition fails although the system is locally weakly observable.

In the following, we argue that a larger number of triads does not change the observability property of the system and thus only adds redundancy. To increase the number of accelerometers we add a fourth triad $T_{4}$ to the original configuration (Table I). The positions of the original triad configuration span a plane. Hence, an additional triad placed on this surface would result in additional acceleration measurements that are linearly dependent on the original ones. Therefore, triad $T_{4}$ is situated out of plane at position $\boldsymbol{r}_{10,11,12}=\left[\begin{array}{lll}0 & 0 & 1\end{array}\right]^{T}$ of the body. Thus, the setup of the sensors complies to the rules derived in [22]. Three sensor equations $h_{10}, h_{11}$, and $h_{12}$ are added to the observation model (12)

$$
\left[\begin{array}{l}
h_{10}(\boldsymbol{x}) \\
h_{11}(\boldsymbol{x}) \\
h_{12}(\boldsymbol{x})
\end{array}\right]=\left[\begin{array}{l}
{ }^{b} a_{x}+\omega_{x} \omega_{z}+\dot{\omega}_{y} \\
{ }^{b} a_{y}+\omega_{y} \omega_{z}-\dot{\omega}_{x} \\
{ }^{b} a_{z}-\omega_{x}^{2}-\omega_{y}^{2}
\end{array}\right] .
$$

Their corresponding Lie-derivatives are introduced to observability matrix $O_{M}$. Again, $O_{M}$ can be partitioned in the same way as in (13). Here, submatrix $A_{6 \times 3}$ is extended by three rows to $A_{9 \times 3}$ and evaluates to

$$
A_{9 \times 3}=\left[\begin{array}{ccc} 
& A_{6 \times 3} & \\
\hline \omega_{z} & 0 & \omega_{x} \\
0 & \omega_{z} & \omega_{y} \\
-2 \omega_{x} & -2 \omega_{y} & 0
\end{array}\right] .
$$

In the same fashion, $B_{6 \times 3}$ is expanded to

$$
B_{9 \times 3}=\left[\begin{array}{rrr} 
& B_{6 \times 3} & \\
\hline 0 & 1 & 0 \\
-1 & 0 & 0 \\
0 & 0 & 0
\end{array}\right] .
$$

As only rows were added to $O_{M}$, its rank will be at least as high, as for the configuration with nine sensors. Thus, it is only necessary to check the rank at points where $O_{M}$ for the nine sensor configuration was rank deficient. If $\boldsymbol{\omega}=\dot{\boldsymbol{\omega}}=\mathbf{0}$, the matrices $A_{9 \times 3}$ and $\dot{A}_{9 \times 3}$ only hold zero entries. As such, also the columns $c_{j}$ with $j=\{4,5,6\}$ of $O_{M}$ only hold zeros. It follows that $O_{M}$ is rank deficient for $\boldsymbol{\omega}=\dot{\boldsymbol{\omega}}=\mathbf{0}$. This is the same result as we obtained for the configuration with three triads. Hence, using more than three triads does not result in a system that shows a different observability characteristic. Instead, the additional transducers only add redundancy, which of course can be used to increase the accuracy of an observer.

\section{State Estimation}

For multiple-input multiple-output systems, the Kalman filter has proven to be an appropriate choice as state observer for a large variety of applications, such as localization [52], orientation estimation [53], or online calibration [54]. Its main advantage is that the state estimation process incorporates the probabilistic nature of the system and the sensor measurements. In this section, we show how to employ a Kalman filter to estimate the state of the GF-IMU system.

The standard Kalman filter supports only linear systems. However, the GF-IMU system is nonlinear in its observation model in (5). To support nonlinear systems, a few extensions of the original Kalman filter have been proposed. The most popular ones are the EKF and the UKF [55]-[57]. In general, the structure of those algorithms is fixed. Only the state definition as well as the process and observation models change for each different application. Thus, in the following, we focus on the models, which are required to employ a nonlinear Kalman filter. The models are not limited to certain variant.

The estimation process of the Kalman filter can be subdivided into two major parts, the prediction and the correction step. The prediction projects the state distribution from the last time step $k-1$ to the current time step $k$. The computations involve the process model $f^{\mathrm{KF}}$, which computes the current state $\boldsymbol{x}_{k}$ based on the state $\boldsymbol{x}_{k-1}$ at the previous time step. It is of the form

$$
\boldsymbol{x}_{k}=\boldsymbol{f}^{\mathrm{KF}}\left(\boldsymbol{x}_{k-1}, \boldsymbol{u}_{k-1}, \boldsymbol{v}_{k-1}\right)
$$

where $\boldsymbol{v}$ is a random variable that represents the noise of the process and $\boldsymbol{u}$ is the input to the system. The correction step generates the estimated state distribution based on the 
predicted state distribution. Here, the observation model $\boldsymbol{h}^{\mathrm{KF}}$ computes the expected measurements. It is of the form

$$
\boldsymbol{y}_{k}=\boldsymbol{h}^{\mathrm{KF}}\left(\boldsymbol{x}_{k}, \boldsymbol{n}_{k}\right)
$$

and takes the state and the noise of the observations $\boldsymbol{n}_{k}$ as input. If the system can be given in the from of $f^{\mathrm{KF}}$ and $\boldsymbol{h}^{\mathrm{KF}}$, basically every kind of nonlinear Kalman filter can be employed.

In order to formulate $f^{\mathrm{KF}}$ we use the original process model $f$ given in (6) and convert it from a continuous time to a discrete time description. However, we do not have access to the input $\boldsymbol{u}$ of the system. Therefore, we model the input of (6) as process noise $\boldsymbol{v}$. For $\boldsymbol{f}^{\mathrm{KF}}$ this yields

$$
\boldsymbol{f}^{\mathrm{KF}}\left(\boldsymbol{x}_{k}, \boldsymbol{v}_{k}\right)=\left[\begin{array}{l}
{ }^{b} \boldsymbol{a}_{k-1}+\boldsymbol{v}_{a} \Delta t \\
\boldsymbol{\omega}_{k-1}+\dot{\boldsymbol{\omega}}_{k-1} \Delta t+\frac{1}{2} \boldsymbol{v}_{\dot{\omega}}(\Delta t)^{2} \\
\dot{\boldsymbol{\omega}}_{k-1}+\boldsymbol{v}_{\dot{\omega}} \Delta t
\end{array}\right]
$$

where $\Delta t$ is the sampling period. The equations of $f^{\mathrm{KF}}$ implement the assumption that between two time steps there is no change in the transversal and angular acceleration. The assumption is qualified by the noise terms $\boldsymbol{v}_{a}$ and $\boldsymbol{v}_{\dot{\omega}}$, which are assumed to be time-independent.

The only change to the observation model $\boldsymbol{h}$ is the incorporation of the observation noise $\boldsymbol{n}$. Hence, for all accelerometer arrays with $m$ transducers the observation model $\boldsymbol{h}^{\mathrm{KF}}$ is given by

$$
\boldsymbol{h}^{\mathrm{KF}}\left(\boldsymbol{x}_{k}, \boldsymbol{n}_{k}\right)=\left[\begin{array}{c}
{ }^{s} a_{1} \\
\vdots \\
{ }^{s} a_{m}
\end{array}\right]+{ }^{s} \boldsymbol{n}
$$

where ${ }^{s} \boldsymbol{n}=\left[{ }^{s} n_{1}, \ldots,{ }^{s} n_{m}\right]^{T}$ is a $m$-dimensional timeindependent vector holding the noise terms of the accelerometer measurements.

The noise terms for the process model $v$ and the observation model $\boldsymbol{n}$ are assumed to be zero-mean normally distributed random variables. Thus, $v$ is drawn from the distribution $\mathcal{N}\left(0, R_{v}\right)$ and $\boldsymbol{n}$ from $\mathcal{N}\left(0, R_{n}\right)$. The process covariance $R_{v}$ is constructed as

$$
R_{v}=\operatorname{diag}\left(\left[\begin{array}{c}
\boldsymbol{\sigma}_{a}^{2} \\
\boldsymbol{\sigma}_{\dot{\omega}}^{2}
\end{array}\right]\right)
$$

where the $3 \times 1$ vectors $\sigma_{a}^{2}$ and $\sigma_{\dot{\omega}}^{2}$ are the variance of the process along each dimension of the body. They express how certain the assumption of constant acceleration holds for both the transversal acceleration with $\sigma_{a}^{2}$ and the angular acceleration with $\sigma_{\dot{\omega}}^{2}$. The observation covariance $R_{n}$ is given by

$$
R_{n}=\operatorname{diag}\left(\left[{ }^{s} \sigma_{1}^{2} \ldots{ }^{s} \sigma_{m}^{2}\right]^{T}\right)
$$

where each entry ${ }^{s} \sigma^{2}$ holds the measurement variance of the respective transducer. These variances can easily be determined by recording a set of steady-state measurements and calculating their variance. In contrast to this, $\sigma_{a}^{2}$ and $\sigma_{\dot{\omega}}^{2}$ can be adjusted such that they best describe the application scenario of the accelerometer array. High values are suitable for motions with large dynamic ranges, whereas low values are appropriate for motions with low dynamic. In our experiments, we observed that the values of $\boldsymbol{\sigma}_{a}^{2}$ and $\boldsymbol{\sigma}_{\dot{\omega}}^{2}$ do not affect the estimation results critically. Still, they can be regarded as tuning parameters of the estimation process.

The models with their respective covariances are suitable for both the EKF and the UKF. The UKF features a good convergence for highly nonlinear systems, whereas the EKF is computationally more efficient [58]. In order to integrate the models into the EKF framework one has to provide the Jacobian matrices of both $\boldsymbol{f}^{\mathrm{KF}}$ and $\boldsymbol{h}^{\mathrm{KF}}$ [55]. In contrast to this, the derived models and their covariances can be applied to the UKF framework without any further changes, which greatly facilitates the model integration.

In our experiments, which we present in the next section, we employ the UKF as observer. We adjusted the process covariance to $\sigma_{a}=\left[\begin{array}{lll}1 & 1 & 1\end{array}\right]^{T} \cdot 750 \mathrm{~m} / \mathrm{s}^{3}$ and $\sigma_{\dot{\omega}}=\left[\begin{array}{lll}1 & 1 & 1\end{array}\right]^{T}$. $750^{\circ} / \mathrm{s}^{3}$, here expressed as standard deviations. Since the Kalman filter recursively estimates the state an initial state $\boldsymbol{x}_{0}$ and its covariance $P_{0}$ must be set. In general, $x_{0}$ is unknown. To account for this, $P_{0}$ should have large values on its main diagonal, since these entries represent the variance of the respective state dimension.

The derived models differ from the ones of our previous works in [38] and [39] by the following. First, they originate from an general system description and are therefore suitable for any observable accelerometer array. Furthermore, as the unknown inputs $\boldsymbol{u}$ are modeled as noise terms $\boldsymbol{v}$, the process model describes the propagation of an angular excitation to the angular acceleration and velocity. Thus, the predicted state distribution now correctly reflects the uncertainty of the constant acceleration assumption.

\section{EXPERIMENTAL RESUlTS}

With the measurements we took on the 3-D rotation table, we aim to demonstrate the estimation behavior of a GF-IMU with two experiments. By means of experiment I, we validate that an accelerometer array of only three triads is capable to estimate an arbitrary motion. Thereby, the estimation accuracy of such an array compares well to arrays that incorporate a higher number of triads. With experiment II, we provide an example that the choice of the initial estimates has an influence on the estimation. This shows, that the GF-IMU system is locally weakly observable but not globally observable.

\section{A. Experimental Setup}

The GF-IMU prototype shown in Fig. 1(b) incorporates five sensor triads in total (Bosch Sensortec, BMA 180). A main board is located at the center of a metal carrier plate, which comprises a microcontroller for data readout and contains one accelerometer triad. Four satellite boards are connected to the main board. Each one of them carries a sensor triad. We determined the positions, orientations, and offsets of each acceleration sensitive axis by applying the calibration method we previously presented in [38]. Fig. 4 shows the placement of the transducers. The sensor positions and sensitivity axes relative to the body frame are summarized in Table II. Although the prototype does not include a configuration of the triads like the one we used for the observability analysis 
(a)

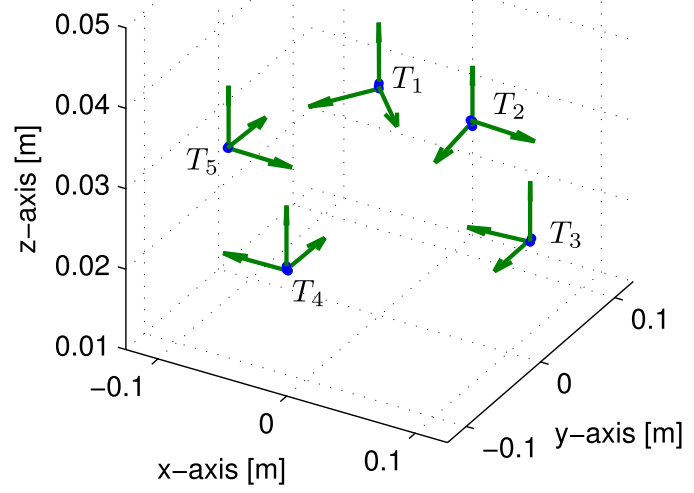

(b)

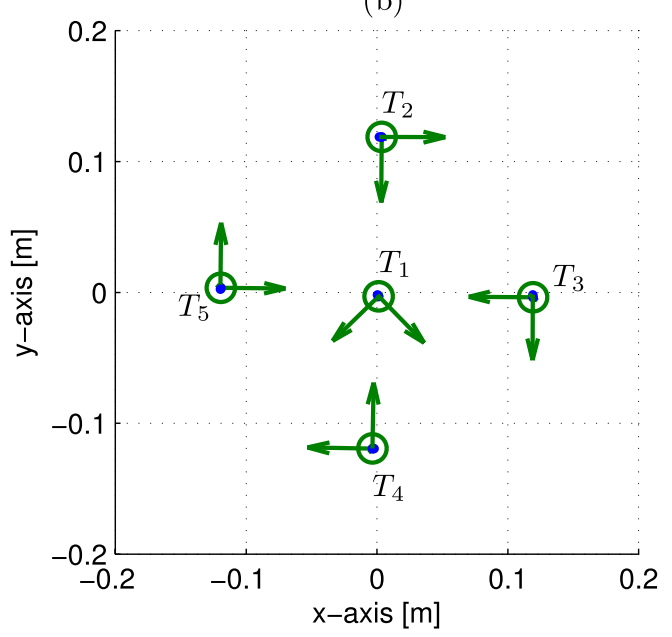

Fig. 4. Positions and orientations of the sensors determined by the calibration shown in (a) perspective view and (b) top view.

it features configurations that are highly related to it, which can be reasoned by the following. For a GF-IMU, the center of the body as well as its orientation can be arbitrarily defined as long as the positions and orientations of the triads are correctly defined relative to the body frame. Furthermore, the single triads can be rotated virtually in the body frame if we apply the same rotation to the measurement vector of the respective triad. Using these transformation, one can generate configurations that are equal to the one we used for the observability analysis except for the scaling of the positions. ${ }^{1}$

The rotation table features a highly accurate measurement instrumentation for the positions of its movable axes. These measurements were used as reference for the motion that was

\footnotetext{
${ }^{1}$ For example, a configuration related to the one shown in Fig. 3 can be generated by the following steps. First, we apply a coordinate transformation of the center of the body to the position of triad $T_{5}$ and then rotate the body about $45^{\circ}$ counterclockwise such that the positions of the two triads $T_{2}$ and $T_{4}$ lie on the major axes of the body. Next, we rotate the triads $T_{2}, T_{4}, T_{5}$ such that their sensitivity axes align with the major axes of the body frame. As a result, the triads $T_{2}, T_{4}, T_{5}$ feature the same configuration as the one used for the observability analysis up to spread of its positions.
}

TABLE II

Positions And Sensitivity AXes of Used Sensors as Determined by Calibration. The 15 Sensors $\left(S_{1}, \ldots, S_{15}\right)$ Are Clustered Into Five SENSOR TRIADS $\left(T_{1}, \ldots, T_{5}\right)$

\begin{tabular}{|c|c|c|c|c|c|c|c|}
\hline \multirow[t]{2}{*}{$T_{j}$} & \multirow[t]{2}{*}{$S_{i}$} & \multicolumn{3}{|c|}{ Position $\boldsymbol{r}_{i}(\mathrm{~cm})$} & \multicolumn{3}{|c|}{ Sensitivity axis $\boldsymbol{s}_{i}$} \\
\hline & & $x$ & $y$ & $z$ & $x$ & $y$ & $z$ \\
\hline \multirow{3}{*}{$T_{1}$} & $S_{1}$ & 0.1 & -0.3 & 3.9 & -0.70 & -0.68 & -0.02 \\
\hline & $S_{2}$ & 0.0 & -0.2 & 3.9 & 0.71 & -0.73 & -0.01 \\
\hline & $S_{3}$ & 0.1 & -0.3 & 3.9 & 0.00 & 0.00 & 1.00 \\
\hline \multirow{3}{*}{$T_{2}$} & $S_{4}$ & 0.4 & 11.9 & 2.5 & -0.01 & -1.00 & -0.02 \\
\hline & $S_{5}$ & 0.2 & 11.9 & 2.5 & 1.01 & 0.00 & -0.01 \\
\hline & $S_{6}$ & 0.4 & 11.9 & 2.4 & 0.00 & -0.01 & 1.00 \\
\hline \multirow{3}{*}{$T_{3}$} & $S_{7}$ & 11.9 & -0.4 & 2.5 & -0.98 & 0.01 & -0.01 \\
\hline & $S_{8}$ & 11.9 & -0.2 & 2.5 & -0.01 & -1.00 & 0.00 \\
\hline & $S_{9}$ & 11.9 & -0.4 & 2.5 & 0.00 & 0.00 & 1.00 \\
\hline \multirow{3}{*}{$T_{4}$} & $S_{10}$ & -0.3 & -11.9 & 2.5 & 0.02 & 1.00 & 0.00 \\
\hline & $S_{11}$ & -0.2 & -11.9 & 2.5 & -1.02 & 0.02 & 0.00 \\
\hline & $S_{12}$ & -0.3 & -12.0 & 2.6 & 0.01 & -0.01 & 1.01 \\
\hline \multirow{3}{*}{$T_{5}$} & $S_{13}$ & -11.9 & 0.4 & 2.5 & 0.99 & -0.01 & 0.00 \\
\hline & $S_{14}$ & -12.0 & 0.2 & 2.5 & 0.01 & 1.01 & 0.00 \\
\hline & $S_{15}$ & -11.9 & 0.3 & 2.5 & 0.00 & -0.01 & 1.00 \\
\hline
\end{tabular}

imposed on the accelerometer array. All measurements were recorded at a sampling frequency of $50 \mathrm{~Hz}$.

For the evaluation of the first experiments, we compare the estimations based on measurements with estimations we obtained using simulated measurement data. Therefore, we generated synthetic measurements by means of the observation model (5) based on the respective reference motion. To simulate the noise of the sensors, we added random numbers to the synthetic measurements drawn from a normal distribution with zero mean and a standard deviation that equals the one we experimentally determined for the sensor triads. We generated the simulated measurements using the same sensor setup as determined by the calibration of the prototype (Table II). Thus, up to minor differences, the simulations represent the same accelerometer array as the prototype but with a perfect knowledge of the positions and sensitive axes of the transducers and without any imperfection of the linear sensor characteristic. The sensor noise is the only external source of errors.

\section{B. Experiment I}

We conducted experiment I according to the following methodology. First, we imposed a rotation $x^{\text {Ref }}$ around all axes on the body and recorded the accelerations measured by all five triads of the prototype. The rotation was designed such that it covers a large area of the state space, i.e., it contains many combinations of ${ }^{b} \boldsymbol{a}, \boldsymbol{\omega}$, and $\dot{\boldsymbol{\omega}}$. Subsequently, we estimated the body motion using the data of three, four, or five triads. This allows us to evaluate ten different configurations of three triads, five configurations of four triads, and one five triad configuration.

Fig. 5 shows $15 \mathrm{~s}$ of the imposed motion. The duration of the complete test run was $120 \mathrm{~s}$. We set the estimate of the initial state to its true value, thus ${ }^{b} \boldsymbol{a}_{0}=\left[\begin{array}{lll}0 & 0 & \mathrm{~g}\end{array}\right]^{T}$ and $\omega_{0}=\dot{\omega}_{0}=\left[\begin{array}{lll}0 & 0 & 0\end{array}\right]^{T}$, with $g$ being the gravitational 
(a)

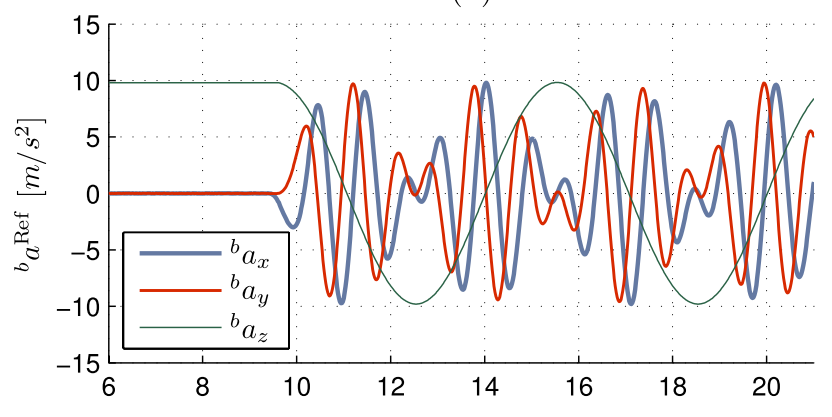

(b)

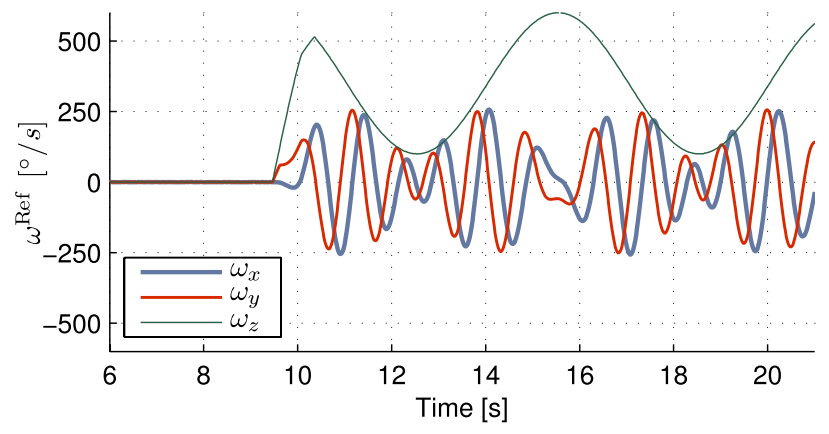

Fig. 5. Motion imposed on the accelerometer array for experiment I showing (a) linear acceleration and (b) angular velocity.

acceleration. However, the choice of the initial state is not crucial for this experiment. As there is no motion imposed for the first seconds the state estimate would also converge to the true state within a few time steps for any other initial state. As a measure of the estimation accuracy at each time step $t$, we used the Euclidean distance between the reference motion $x^{\text {Ref }}$ and the estimated motion $\hat{\boldsymbol{x}}$. We evaluated the Euclidean distances $\left\|\left.\right|^{b} \boldsymbol{a}^{\mathrm{Ref}}-{ }^{b} \hat{\boldsymbol{a}}\right\|_{t}$ and $\left\|\boldsymbol{\omega}^{\mathrm{Ref}}-\hat{\boldsymbol{\omega}}\right\|_{t}$ separately for both the linear acceleration and the angular velocity according to

$$
\left\|\boldsymbol{p}^{\mathrm{Ref}}-\hat{\boldsymbol{p}}\right\|_{t}=\sqrt{\left(\boldsymbol{p}_{t}^{\mathrm{Ref}}-\hat{\boldsymbol{p}}_{t}\right)^{T}\left(\boldsymbol{p}_{t}^{\mathrm{Ref}}-\hat{\boldsymbol{p}}_{t}\right)}
$$

with $\boldsymbol{p}$ being either ${ }^{b} \boldsymbol{a}$ or $\boldsymbol{\omega}$. To assess the estimation accuracy of the entire test run, we used the mean $\overline{\left\|p^{\text {Ref }}-\hat{\boldsymbol{p}}\right\|}$ of the Euclidean distances of all time steps.

Table III summarizes the mean Euclidean distances of all possible triad configurations. The configurations are sorted according to the resulting mean Euclidean distances $\overline{\left\|\omega^{\mathrm{Ref}}-\hat{\boldsymbol{\omega}}\right\|}$ of the simulations. Looking at this order, two influences that have an impact on the estimation errors can be observed. First, configurations that contain a larger number of triads have a smaller error. This is reasonable because the additional sensors provide extra information to estimate the angular motion. Second, for configurations with the same number of triads the estimation accuracy depends on the placement of the sensors. For example, configuration 4.1 yields the smallest error of all configurations with four triads. From a top view perspective, the triad positions of this configuration enclose the largest area as any other configuration of four triads includes $T_{1}$. The same can be observed for configurations using three triads. Here, 3.1-3.4 do not include $T_{1}$
TABLE III

Mean Euclidean Distances Between the Reference Motion $\boldsymbol{x}^{\text {Ref }}$ And the Estimated Motion $\hat{\boldsymbol{x}}$ of Experiment I of All Possible Configurations of Three, Four, or Five ACCELEROMETER TRIADS FOR BOTH EXPERIMENTAL (EXP.) AND Simulated (Sim.) DATA

\begin{tabular}{|c|c|c|c|c|c|}
\hline \multicolumn{2}{|c|}{ Configuration } & \multicolumn{2}{|c|}{$\begin{array}{c}\overline{\| b} \boldsymbol{a}^{\operatorname{Ref}}-{ }^{b} \hat{\boldsymbol{a}} \| \\
\text { in } \mathrm{mg} / \mathrm{s}^{2}\end{array}$} & \multicolumn{2}{|c|}{$\begin{array}{c}\overline{\left\|\boldsymbol{\omega}^{\mathrm{Ref}}-\hat{\boldsymbol{\omega}}\right\|} \\
\text { in } \% / \mathrm{s}\end{array}$} \\
\hline No. & Used triads & Sim. & Exp. & Sim. & Exp. \\
\hline 5.1 & $\{1,2,3,4,5\}$ & 2.44 & 8.50 & 1.71 & 4.10 \\
\hline 4.1 & $\{2,3,4,5\}$ & 2.45 & 8.51 & 1.84 & 4.29 \\
\hline 4.2 & $\{1,2,3,5\}$ & 3.82 & 9.68 & 2.01 & 4.67 \\
\hline 4.3 & $\{1,2,4,5\}$ & 3.70 & 9.55 & 2.05 & 4.16 \\
\hline 4.4 & $\{1,3,4,5\}$ & 3.94 & 9.64 & 2.05 & 5.97 \\
\hline 4.5 & $\{1,2,3,4\}$ & 3.85 & 9.61 & 2.08 & 5.08 \\
\hline 3.1 & $\{2,3,5\}$ & 4.67 & 10.07 & 2.16 & 4.60 \\
\hline 3.2 & $\{2,4,5\}$ & 4.22 & 10.05 & 2.26 & 4.97 \\
\hline 3.3 & $\{3,4,5\}$ & 4.71 & 9.99 & 2.31 & 5.50 \\
\hline 3.4 & $\{2,3,4\}$ & 4.27 & 9.90 & 2.36 & 4.27 \\
\hline 3.5 & $\{1,3,4\}$ & 7.92 & 13.44 & 2.47 & 8.43 \\
\hline 3.6 & $\{1,2,5\}$ & 7.24 & 12.77 & 2.66 & 4.96 \\
\hline 3.7 & $\{1,2,3\}$ & 7.49 & 13.19 & 2.79 & 8.55 \\
\hline 3.8 & $\{1,4,5\}$ & 7.72 & 12.95 & 3.20 & 7.12 \\
\hline 3.9 & $\{1,2,4\}$ & 21.69 & 26.91 & 21.37 & 26.34 \\
\hline 3.10 & $\{1,3,5\}$ & 21.46 & 25.94 & 22.14 & 24.60 \\
\hline
\end{tabular}

and therefore their positions span a triangle which is larger than any other configuration of three triads which is why they yield the smallest error. The triad positions of 3.9 and 3.10 do not form a triangle from a top view perspective. Instead, they approximately reside on a line. However, $T_{1}$ is situated above the other triads (Table II) and thus the triads span a triangle from a side view perspective. The enclosed area is small compared with the other configurations due to the small relative height of $T_{1}$. As a result, the estimation accuracy of 3.9 and 3.10 is poor in proportion to other configurations. The above rationale clusters the configurations into six groups with the same number of triads and a similar arrangement of the transducers in terms of the enclosed area. The groups are indicated by the additional horizontal lines in Table III.

The mean Euclidean distances $\overline{\left\|^{b} \boldsymbol{a}^{\mathrm{Ref}}-{ }^{b} \hat{\boldsymbol{a}}\right\|}$ of the simulations confirm the clustering of the configurations. If the estimation of the angular velocity is erroneous, the estimation of the linear acceleration contains errors as well. Thus, estimation errors do not appear on only a single state dimension. Instead, they are distributed among all state variables. With simulated data, the number of triads and their configuration seem to have a stronger impact on the accuracy of the linear acceleration as on the accuracy of the angular velocity. However, this effect heavily depends on the variances $\sigma_{a}^{2}$ and $\sigma_{\dot{\omega}}^{2}$ of $R_{v}$ that define how much the estimation process relies on the assumption of constant acceleration for either the linear or the angular acceleration. Higher values of the variances for a certain state variable account for a more dynamical estimation of this dimension. Thus, the state variable is more likely affected by sensor noise. The order of within each group of configurations is not be the same for $\overline{\left\|^{b} \boldsymbol{a}^{\mathrm{Ref}}-{ }^{b} \hat{\boldsymbol{a}}\right\|}$ 
and $\overline{\left\|\omega^{\text {Ref }}-\hat{\boldsymbol{\omega}}\right\|}$, which can be reasoned by the following. The noise of the measurements leads to a false estimation of the motion, which is distributed among the state variables. Whether this has more effect on the linear acceleration or the angular velocity depends on the minor geometry differences and on the random measurement error.

The evaluations with experimental data are in good accordance to the evaluation using simulated measurements. However, the error of the experiments is higher than the error of the simulations. We reason this by the following. In contrast to the simulation, the estimation with experimental data contains errors in the sensor description. Those are, for example, an inaccurate knowledge of the sensor parameters, i.e., its position, orientation, or offset, which result from an erroneous calibration. Furthermore, sensor nonidealities, such as nonlinearity, temperature drift, or cross coupling are not covered by the linear sensor model. If such flaws occur within the description of the accelerometers array the predicted measurements contain a systematic error. This distorts the estimated motion. Based on our experience with the GF-IMU prototype, we believe that the main source of additional errors compared with the simulation is an imperfect calibration of the sensors.

In summary, experiment I verifies that accelerometer arrays with three sensor triads can be utilized to estimate an arbitrary spatial motion. Furthermore, such configurations feature a comparable performance to arrays with a higher number of triads. However, the positions of the sensors must span a surface regardless of the number of triads. The simulation and experimental results indicate that the estimation accuracy depends on the area enclosed by the positions of the sensor triads. However, experiment I together with the employed sensor configurations does not provide a performance evaluation for the number of employed triads given the best possible sensor locations. A more extensive analysis of this relationship necessary to derive a general conclusion will be part of our future research. An interesting approach to the efficient placement under geometrical constraints of the transducers can be found in [15]. However, we are solely concerned with an indirect approach and therefore do not consider the angular velocity.

\section{Experiment II}

In order to provide an example of the GF-IMU being locally weakly observable we evaluate the same measurement data in two estimation runs. Those only differ in the choice of the initial state $\hat{\boldsymbol{x}}_{0}$. Here, we present only the results obtained with measurement data, as the simulation resulted in a very similar behavior that differed only in terms of accuracy. Furthermore, we consider only configuration 3.4 of Table III since this configuration incorporates the minimum number of three triads and showed the best experimental accuracy for the angular velocity in experiment I.

To reduce the complexity of the evaluation we impose a simple 1-D rotation around the $z$-axis on the accelerometer array shown in Fig. 6. The peaks in the estimated angular acceleration originate from a disorder in the control loop of the rotation table, which generates a small imbalance of the

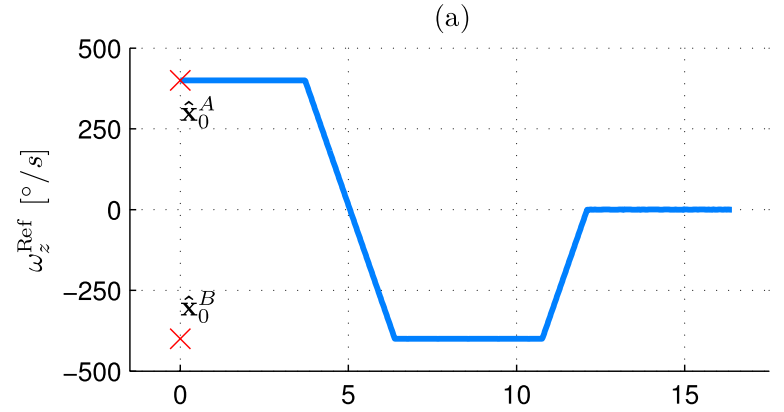

(b)

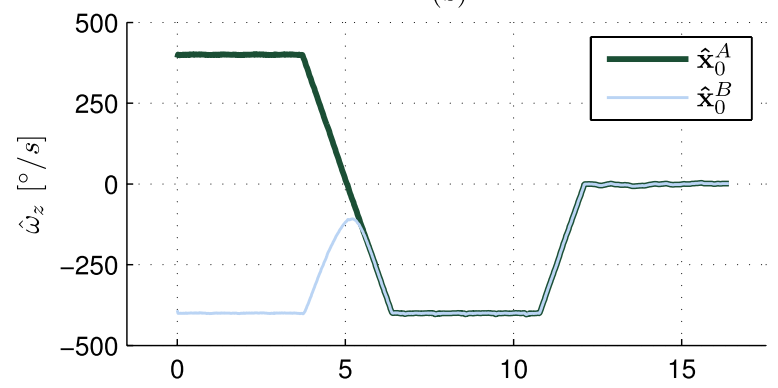

(c)

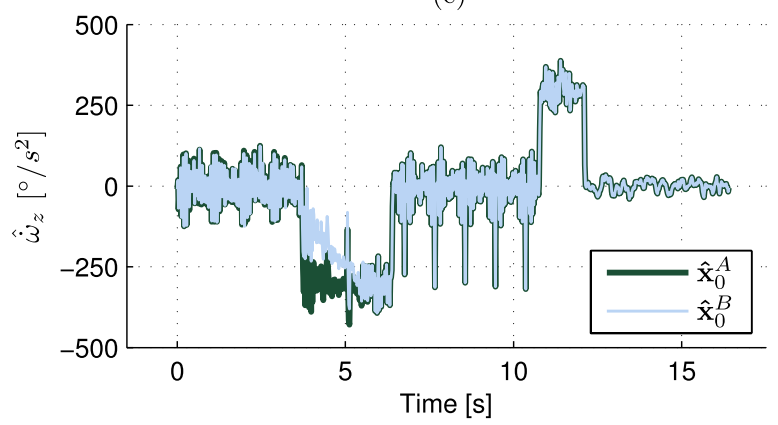

Fig. 6. Estimated angular velocity for the rotation around the body's $z$-axis of experiment II using the three triad configuration 3.4. (a) Imposed rotation is shown, while (b) and (c) depict the estimated angular velocity and acceleration.

imposed angular velocity. However, the recorded motion is not affected by this.

The estimation starts at a time when the accelerometer array is already rotating. The two different initial states $\hat{\boldsymbol{x}}_{0}^{A}$ and $\hat{\boldsymbol{x}}_{0}^{B}$ are set to the amplitude of the reference state provided by the table but with opposite signs for the angular velocity $\omega_{z}$ [Fig. 6(a)]. Until the angular velocity starts changing the UKF correctly estimates the amplitude of $\omega_{z}$, which can be seen in Fig. 6(b). However, the signs of the estimated rotations remain opposite as predetermined by their corresponding initial values. This can be reasoned by the information that is available for the state estimation. Only a constant rotation is imposed on the GF-IMU. As such, the motion only excites a transversal acceleration ${ }^{b} \boldsymbol{a}$ and a centrifugal acceleration $\boldsymbol{\omega} \times(\boldsymbol{\omega} \times \boldsymbol{r})$ as stated by (1). Therefore, the estimates of both initial values converge to the correct magnitude of the angular velocity. However, because of the quadratic dependency between the angular velocity and the centrifugal acceleration the measured accelerations are independent of the direction of the imposed rotation. Due to this symmetry within the 
observation model the GF-IMU system is not globally observable. If only a constant rotation is present, positive and negative rotations cannot be distinguished regardless of the duration of the rotation.

After the period of constant angular velocity, a negative angular acceleration is imposed on the GF-IMU. Since the applied angular velocity is decreasing, the accelerometers are excited by two different forces: a decreasing centrifugal acceleration $\boldsymbol{\omega} \times(\boldsymbol{\omega} \times \boldsymbol{r})$ due to the declining absolute value of $\omega_{z}$ and a constant negative tangential acceleration $\dot{\omega} \times \boldsymbol{r}$ caused by the negative $\dot{\omega}_{z}$. For the evaluation with initial state $\hat{\boldsymbol{x}}_{0}^{A}$, these two accelerations are consistent with the current estimated angular velocity and hence the UKF correctly tracks the reference motion. However, they are contrary for the evaluation with initial state $\hat{\boldsymbol{x}}_{0}^{B}$ since a decreasing absolute value of a negative angular velocity implies a positive angular acceleration. This relation is described within the Kalman filter by process model $f^{\mathrm{KF}}$. Hence, the combination of the conflicting angular velocity estimate and acceleration measurements leads to incorrect state estimations for the evaluation with initial state $\hat{\boldsymbol{x}}_{0}^{B}$. For example, the angular acceleration $\dot{\omega}_{z}$ is distorted to higher values than originally imposed on the GF-IMU [Fig. 6(c)].

As the estimates $\hat{\omega}_{z}$ of the angular velocity intersect, both correctly follow the reference values [Fig. 6(b)]. This exemplarily shows that the GF-IMU system is locally weakly observable. Once the state estimate coincides with the true motion, the UKF properly tracks the sign and the amplitude of the angular velocity. The predicted measurements are now consistent with the actual measurements.

During the first phase of constant rotation, experiment II demonstrates that the angular velocity is in direct relation to the centrifugal acceleration. For any initial state, the centrifugal acceleration causes the estimate to converge to the correct magnitude of the rotation while the direction is predetermined by the sign of the initial state. However, in most application scenarios, the estimation starts while the body is at rest just as the motion of experiment I. In this case, there is no centrifugal acceleration present and hence the estimated angular velocity of any initial state converges to zero within only a few time-steps. Thus, the estimated and the true state coincide, which enables the Kalman filter to correctly track the motion.

Experiment II also illustrates a deficiency of the Kalman filter approach. After a first period of constant rotation there is basically sufficient information available to establish a correct estimation of the magnitude and the direction. The estimate with initial state $\hat{\boldsymbol{x}}_{0}^{B}$ is not plausible as it is not consistent with the measurement. However, the UKF does not switch to the correct angular velocity because this sign change is unlikely considering the estimated covariance $P_{k}$. This drawback results from the assumption that the state is normally distributed around its mean, which is made for all Kalman filters. As such only unimodal probability distributions are well represented. However, if only the measurements recorded at the period of constant rotation are taken into account, both a positive and a negative rotation are equally likely. As such, the state features a multimodal distribution. There are probabilistic filters that support such a state distribution, e.g., the histogram or the particle filter [57]. However, these introduce other severe drawbacks when applied as GF-IMU observer. For example, the histogram filter is limited to the accuracy of a predefined grid spanned over the state space whereas the particle filter demands large computational resources due to the high dimensionality of the defined state. Still, these are interesting approaches that might be applicable in a hybrid combination with the Kalman filter. As such, the presented issue as well as investigation of alternative observer approaches is part of our future research.

\section{COnClusion}

In this paper, we are concerned with a GF-IMU that is based on nine accelerometers. We show that in general such a sensor array is capable to directly capture an arbitrary spatial motion and in particular the angular velocity. First, we model the GF-IMU as a control system. This enables us to conduct an analytical observability analysis that is based on the concept of LWO. In order to restrict the analysis to reasonable array configurations we introduce constraints on the placement of the transducers, which are justified by nowadays commercially available triaxial accelerometers. The constraints imply that the sensors are grouped into three sensor triads and their positions span a surface. This greatly reduces the complexity of the analysis while remaining sufficiently general. The main result of this analysis is that the GF-IMU system is locally weakly observable for every motion except for its equilibrium point where no angular motion is present. However, this point is identifiable. Hence, it is possible to estimate the relative motion using an array of three sensor triads. Any additional triads only provide redundant information.

The derived system description allows for a simple integration of the process and observation models into the Kalman filter framework. Only a continuous to discrete time conversion of the process model and the extension of both models by the appropriate noise terms is necessary. As the established models are of a general form, the presented Kalman filter approach is suitable for any observable GF-IMU. Furthermore, they can be applied to any nonlinear Kalman filter variant.

With experiments taken on a 3-D rotation table we illustrate the estimation behavior of a GF-IMU. First, we compare the estimation accuracy of multiple three triad configurations against configurations of four and five triads. The evaluations confirm the results of the observability analysis. In addition, redundant sensors can be employed to yield a higher estimation accuracy. Furthermore, we provide an explanatory experiment, which illustrates the property of LWO of the GFIMU system. The experiment also demonstrates a deficiency of the Kalman filter approach that occurs in case the accelerometer array is already in a constant rotation at the start of the estimation and the initial estimate is set to the wrong direction of the rotation. Here, the Kalman filter estimate does not correspond to the true motion until both intersect for the first time although there is appropriate measurement information available beforehand. In our future research, we 
will therefore investigate how we can extend the current approach to eliminate this shortcoming while retaining its computational efficiency.

\section{APPENDIX}

\section{LINEAR INDEPENDENCE OF THE COLUMNS OF $O_{M}$}

We subdivide the problem into the following parts. First, we prove that the columns of the three groups of columns $c_{i}$ with $i=\{1,2,3\}, i=\{4,5,6\}$, and $i=\{7,8,9\}$ are linearly independent within each group. Then, we show for every group, that no linear combination of columns, which are not in that group, can give one of its columns.

Columns $i=\{1,2,3\}$ : Consider the first three rows $r_{j}$ with $j=\{1,2,3\}$. Within these rows, the columns $i=\{1,2,3\}$ contain an identity matrix. Thus, the first three columns of $O_{M}$ with $i=\{1,2,3\}$ are linearly independent among each other.

Columns $i=\{4,5,6\}$ : In order to determine the number of linearly independent columns with $i=\{4,5,6\}$ we first check if matrix $A_{6 \times 3}$ has rank 3 and hence has three linearly independent columns. However, $A_{6 \times 3}$ is not a square matrix. Thus, we first compute its Gramian $A^{T} A$, which naturally has the same rank as $A_{6 \times 3}$. Since $A^{T} A$ is a square matrix its determinant is zero if $A^{T} A$ is not full rank. For matrix $A_{6 \times 3}$ the determinant $\operatorname{det}\left(A^{T} A\right)$ of its Gramian yields to

$$
\begin{aligned}
\operatorname{det}\left(A^{T} A\right)= & 8\left(\omega_{x}^{2}+\omega_{y}^{2}\right)^{3}+20\left(\omega_{x}^{2}+\omega_{y}^{2}\right)^{2} \omega_{z}^{2} \\
& +24\left(\omega_{x}^{2}+\omega_{y}^{2}\right) \omega_{z}^{4}+8 \omega_{z}^{6}
\end{aligned}
$$

which evaluates to zero only when $\omega_{x}=\omega_{y}=\omega_{z}=0$. Thus, $A_{6 \times 3}$ is of full rank for any $\boldsymbol{x}$ but for the case when no angular velocity is present. For this case, we also must consider the rank of matrix $\dot{A}_{6 \times 3} . \dot{A}_{6 \times 3}$ has the same structure as $A_{6 \times 3}$ with the entries being the time derivatives of the angular velocity. Hence, the $\operatorname{determinant} \operatorname{det}\left(\dot{A}^{T} \dot{A}\right)$ is zero only when $\dot{\omega}_{x}=\dot{\omega}_{y}=\dot{\omega}_{z}=0$. By combining the two results, we can make the following statement: the group of columns $i \in\{4,5,6\}$ is linearly independent for all $\boldsymbol{x}$, but for the case when the angular acceleration and velocity is zero $(\omega=\dot{\omega}=\mathbf{0})$. In this special case, the columns $i=\{4,5,6\}$ are zero vectors.

Columns $i=\{7,8,9\}$ : In order to show that the columns with $i=\{7,8,9\}$ are linearly independent, we check if the matrix $B_{6 \times 3}$ is of rank 3 . We apply the same formalism as for the group of columns with $i=\{4,5,6\}$. Since $\operatorname{det}\left(B^{T} B\right)=2$ matrix $B$ is of rank 3 . Therefore, the columns $i \in\{7,8,9\}$ are linearly independent among each other for every motion $\boldsymbol{x}$.

We have now shown that within the three groups the columns are linearly independent. At rows $j=\{1,2,3\}$ the columns $i=\{4, \ldots, 9\}$ of $O_{M}$ only contain zero entries. Hence, any of the first three columns $i=\{1,2,3\}$ of $O_{M}$ can not be given by a linear combination of any other columns $i=\{4, \ldots, 9\}$ and a linear combination of $i \in\{1,2,3\}$ cannot give any of the columns $i=\{4, \ldots, 9\}$. Therefore, the columns $c_{i}$ with $i \in\{1,2,3\}$ do not need to be considered any further within the rank determination.

The remaining problem is to prove that no linear combination of columns with $i=\{4,5,6\}$ can give a column with $i \in\{7,8,9\}$ and vice versa. The following rationale for this is based on the structure of $O_{M}$. In order to check if a linear combination of the columns $i=\{4,5,6\}$ can give any column $i=\{7,8,9\}$ we consider the last three rows of $O_{M}(\boldsymbol{x})$ with $j=\{16,17,18\}$. Clearly, the columns of the zero entry matrix $0_{6 \times 3}$ cannot give a linear combination for the columns of $2 \dot{A}_{6 \times 3}$. Thus, the columns $i=\{4,5,6\}$ are linearly independent of the columns $i=\{7,8,9\}$. However, this statement only holds true as long as $\dot{\boldsymbol{\omega}} \neq \mathbf{0}$, which can be seen by looking at the entries of $2 \dot{A}_{6 \times 3}$. Every column contains every entry of $\dot{\boldsymbol{\omega}}$. Thus, only for the case when $\dot{\boldsymbol{\omega}}=\mathbf{0}$ matrix $\dot{A}_{6 \times 3}$ becomes $0_{6 \times 3}$. Now, the problem is shifted to the rows $j=\{13,14,15\}$. Since $\dot{A}_{6 \times 3}=0_{6 \times 3}$, columns $i=\{4,5,6\}$ are linear independent from the columns $i=\{7,8,9\}$ due to the occupied entries of $A_{6 \times 3}$. However, its columns are zero vectors if $\boldsymbol{\omega}=\mathbf{0}$. Thus, the columns of the two groups $i=\{4,5,6\}$ and $i=\{7,8,9\}$ are linearly independent for all $\boldsymbol{x}$ but for the case when $\boldsymbol{\omega}=\dot{\boldsymbol{\omega}}=\mathbf{0}$.

We can now conclude that $O_{M}$ is of full rank for all $\boldsymbol{x}$ but for the case when $\boldsymbol{\omega}=\dot{\boldsymbol{\omega}}=\mathbf{0}$.

\section{REFERENCES}

[1] N. El-Sheimy, K.-W. Chiang, and A. Noureldin, "The utilization of artificial neural networks for multisensor system integration in navigation and positioning instruments," IEEE Trans. Instrum. Meas. vol. 55 , no. 5, pp. 1606-1615, Oct. 2006.

[2] F. Jiancheng and Y. Sheng, "Study on innovation adaptive EKF for in-flight alignment of airborne POS," IEEE Trans. Instrum. Meas., vol. 60 , no. 4, pp. 1378-1388, Apr. 2011.

[3] D. A. Grejner-Brzezinska, C. K. Toth, H. Sun, X. Wang, and C. Rizos, "A robust solution to high-accuracy geolocation: Quadruple integration of GPS, IMU, pseudolite, and terrestrial laser scanning," IEEE Trans. Instrum. Meas., vol. 60, no. 11, pp. 3694-3708, Nov. 2011.

[4] L. Li et al., "Cart-mounted geolocation system for unexploded ordnance with adaptive ZUPT assistance," IEEE Trans. Instrum. Meas., vol. 61, no. 4, pp. 974-979, Apr. 2012

[5] F. Höflinger, J. Müller, R. Zhang, L. M. Reindl, and W. Burgard, "A wireless micro inertial measurement unit (IMU)," IEEE Trans. Instrum. Meas., vol. 62, no. 9, pp. 2583-2595, Sep. 2013.

[6] O. Bebek et al., "Personal navigation via high-resolution gait-corrected inertial measurement units," IEEE Trans. Instrum. Meas., vol. 59, no. 11, pp. 3018-3027, Nov. 2010.

[7] A. R. J. Ruiz, F. S. Granja, J. C. Prieto Honorato, and J. I. G. Rosas, "Accurate pedestrian indoor navigation by tightly coupling foot-mounted IMU and RFID measurements," IEEE Trans. Instrum. Meas., vol. 61, no. 1, pp. 178-189, Jan. 2012.

[8] G. Panahandeh, N. Mohammadiha, A. Leijon, and P. Handel, "Continuous hidden Markov model for pedestrian activity classification and gait analysis," IEEE Trans. Instrum. Meas., vol. 62, no. 5, pp. 1073-1083, May 2013.

[9] C. Nam, H. Kang, and Y. Suh, "Golf swing motion tracking using inertial sensors and a stereo camera," IEEE Trans. Instrum. Meas., vol 63, no. 4 pp. 943-952, Apr. 2014.

[10] N. Barbour and G. Schmidt, "Inertial sensor technology trends," IEEE Sensors J., vol. 1, no. 4, pp. 332-339, Dec. 2001.

[11] N. Yazdi, F. Ayazi, and K. Najafi, "Micromachined inertial sensors," Proc. IEEE, vol. 86, no. 8, pp. 1640-1659, Aug. 1998.

[12] M. Trächtler, T. Link, J. Dehnert, J. Auber, P. Nommensen, and Y. Manoli, "Novel 3-axis gyroscope on a single chip using SOI-technology," in Proc. IEEE Sensors, Oct. 2007, pp. 124-127.

[13] T. Northemann, M. Maurer, S. Rombach, A. Buhmann, and Y. Manoli, "A digital interface for gyroscopes controlling the primary and secondary mode using bandpass sigma-delta modulation," Sens. Actuators A, Phys., vol. 162, no. 2, pp. 388-393, Aug. 2010.

[14] S. J. Ovaska and S. Valiviita, "Angular acceleration measurement: A review," IEEE Trans. Instrum. Meas., vol. 47, no. 5, pp. 1211-1217, Oct. 1998.

[15] W. T. Latt, U. Tan, C. N. Riviere, and W. T. Ang, "Placement of accelerometers for high sensing resolution in micromanipulation," Sens. Actuators A, Phys., vol. 167, no. 2, pp. 304-316, Mar. 2011. 
[16] J. A. Newman, M. C. Beusenberg, N. Shewchenko, C. Withnall, and E. Fournier, "Verification of biomechanical methods employed in a comprehensive study of mild traumatic brain injury and the effectiveness of American football helmets," J. Biomech., vol. 38, no. 7, pp. 1469-1481, Jul. 2005.

[17] P. Cappa, F. Patanè, and S. Rossi, "A redundant accelerometric cluster for the measurement of translational and angular acceleration and angular velocity of the head," J. Med. Devices, vol. 1, no. 1, pp. 14-22, Aug. 2007.

[18] N. K. Mital and A. I. King, "Computation of rigid-body rotation in threedimensional space from body-fixed linear acceleration measurements," J. Appl. Mech., vol. 46, no. 4, pp. 925-930, Dec. 1979.

[19] N. Yoganandan, J. Zhang, F. A. Pintar, and Y. K. Liu, "Lightweight low-profile nine-accelerometer package to obtain head angular accelerations in short-duration impacts," J. Biomech., vol. 39, no. 7, pp. 1347-1354, Mar. 2006.

[20] Y. K. Peng and M. F. Golnaraghi, "A vector-based gyro-free inertial navigation system by integrating existing accelerometer network in a passenger vehicle," in Proc. IEEE/ION Posit., Location, Navigat. Symp., Apr. 2004, pp. 234-242.

[21] A. Buhmann, C. Peters, M. Cornils, and Y. Manoli, "A GPS aided full linear accelerometer based gyroscope-free navigation system," in Proc. IEEE/ION Posit., Location, Navigat. Symp., Apr. 2006, pp. 622-629.

[22] B. Zappa, G. Legnana, A. J. van den Bogert, and R. Adamini, "On the number and placement of accelerometers for angular velocity and acceleration determination," J. Dyn. Syst., Meas., Control, vol. 123, no. 3, pp. 552-554, Sep. 2001.

[23] J. R. W. Morris, "Accelerometry-A technique for the measurement of human body movements," J. Biomech., vol. 6, no. 6, pp. 729-736, Nov. 1973.

[24] A. J. Padgaonkar, K. W. Krieger, and A. I. King, "Measurement of angular acceleration of a rigid body using linear accelerometers," J. Appl. Mech., vol. 42, no. 3, pp. 552-556, Sep. 1975.

[25] D. H. Wang and G. Yuan, "A six-degree-of-freedom acceleration sensing method based on six coplanar single-axis accelerometers," IEEE Trans. Instrum. Meas., vol. 60, no. 4, pp. 1433-1442, Apr. 2011.

[26] J.-H. Chen, S.-C. Lee, and D. B. Debra, "Gyroscope free strapdown inertial measurement unit by six linear accelerometers," J. Guid., Control, Dyn., vol. 17, no. 2, pp. 286-290, Mar. 1994.

[27] C.-W. Tan and S. Park, "Design of accelerometer-based inertial navigation systems," IEEE Trans. Instrum. Meas., vol. 54, no. 6 , pp. 2520-2530, Dec. 2005.

[28] J. Genin, J. Hong, and W. Xu, "Accelerometer placement for angular velocity determination," J. Dyn. Syst., Meas., Control, vol. 119, no. 3, pp. 474-477, Sep. 1997.

[29] P. Cardou, "Computing the rigid-body acceleration field from nine accelerometer measurements," in Proc. 25th Int. Symp. Brain, Body Mach., Nov. 2010, pp. 325-339.

[30] J. P. B. Vreeburg, "Prony analysis of flat-spin motion from accelerometer data," IEEE Trans. Aerosp. Electron. Syst., vol. 48, no. 2, pp. 1080-1099, Apr. 2012.

[31] S. Park, C.-W. Tan, and J. Park, "A scheme for improving the performance of a gyroscope-free inertial measurement unit," Sens. Actuators A, Phys., vol. 121, no. 2, pp. 410-420, Jun. 2005

[32] T.-L. Chen and S. Park, "MEMS SoC: Observer-based coplanar gyro-free inertial measurement unit," J. Micromech. Microeng., vol. 15, no. 9, pp. 1664-1673, Jul. 2005.

[33] P. Cappa, L. Masia, and F. Patanè, "Numerical validation of linear accelerometer systems for the measurement of head kinematics," J. Biomech. Eng., vol. 127, no. 6, pp. 919-928, Jul. 2005.

[34] K. Parsa, J. Angeles, and A. K. Misra, "Rigid-body pose and twist estimation using an accelerometer array," Archive Appl. Mech., vol. 74 , nos. 3-4, pp. 223-236, Dec. 2004.

[35] K. Parsa, T. A. Lasky, and B. Ravani, "Design and implementation of a mechatronic, all-accelerometer inertial measurement unit," IEEE/ASME Trans. Mechatronics, vol. 12, no. 6, pp. 640-650, Dec. 2007.

[36] C.-W. Ho and P.-C. Lin, "Design and implementation of a 12-axis accelerometer suite," in Proc. IEEE/RSJ Int. Conf. Intell. Robots Syst. (IROS), Oct. 2009, pp. 2197-2202.

[37] J.-C. Lu and P.-C. Lin, "State derivation of a 12-axis gyroscope-free inertial measurement unit," Sensors, vol. 11, no. 3, pp. 3145-3162, Mar. 2011

[38] P. Schopp, L. Klingbeil, C. Peters, and Y. Manoli, "Design, geometry evaluation, and calibration of a gyroscope-free inertial measurement unit," Sens. Actuators A, Phys., vol. 162, no. 2, pp. 379-387, Aug. 2010.
[39] P. Schopp, A. Rottmann, L. Klingbeil, W. Burgard, and Y. Manoli, "Gaussian process based state estimation for a gyroscope-free IMU," in Proc. IEEE Sensors, Nov. 2010, pp. 873-878.

[40] E. Edwan, S. Knedlik, and O. Loffeld, "Constrained angular motion estimation in a gyro-free IMU," IEEE Trans. Aerosp. Electron. Syst., vol. 47, no. 1, pp. 596-610, Jan. 2011.

[41] S. Park and S. K. Hong, "Angular rate estimation using a distributed set of accelerometers," Sensors, vol. 11, no. 11, pp. 10444-10457, Nov. 2011.

[42] P. S. Maybeck, Stochastic Models, Estimation and Control, vol. 1. New York, NY, USA: Academic, 1979.

[43] Y. Bar-Shalom, X. R. Li, and T. Kirubarajan, Estimation with Applications to Tracking and Navigation. New York, NY, USA: Wiley, 2001.

[44] R. Hermann and A. J. Krener, "Nonlinear controllability and observability," IEEE Trans. Autom. Control, vol. 22, no. 5, pp. 728-740, Oct. 1977.

[45] A. Martinelli and R. Siegwart, "Observability analysis for mobile robot localization," in Proc. IEEE/RSJ Int. Conf. IROS, Aug. 2005, pp. 1471-1476.

[46] J. Wu, M. Goldfarb, and E. Barth, "On the observability of pressure in a pneumatic servo actuator," J. Dyn. Syst., Meas., Control, vol. 126, no. 4 pp. 921-924, Mar. 2005.

[47] Y.-B. Jia and M. Erdmann, "Pose and motion from contact," Int. J. Robot. Res., vol. 18, no. 5, pp. 466-487, May 1999.

[48] F. M. Mirzaei and S. I. Roumeliotis, "A Kalman filter-based algorithm for IMU-camera calibration: Observability analysis and performance evaluation," IEEE Trans. Robot., vol. 24, no. 5, pp. 1143-1156, Oct. 2008.

[49] H. Nijmeijer and A. van der Schaft, Nonlinear Dynamical Control Systems. New York, NY, USA: Springer-Verlag, 1990.

[50] A. Isidori, Nonlinear Control Systems. New York, NY, USA: Springer-Verlag, 1995.

[51] M. Anguelova, "Observability and identifiability of nonlinear systems with applications in biology," Ph.D. dissertation, Dept. Math. Sci., Chalmers Univ. Technol., Göteborg, Sweden, 2007.

[52] R. Zhang, F. Höflinger, and L. Reindl, "TDOA-based localization using interacting multiple model estimator and ultrasonic transmitter/receiver," IEEE Trans. Instrum. Meas., vol. 62, no. 8, pp. 2205-2214, Aug. 2013.

[53] E. Kraft, "A quaternion-based unscented Kalman filter for orientation tracking," in Proc. 6th Int. Conf. Inform. Fusion, vol. 1. Jul. 2003, pp. $47-54$.

[54] M. Glück, D. Oshinubi, P. Schopp, and Y. Manoli, "Real-time autocalibration of MEMS accelerometers," IEEE Trans. Instrum. Meas., vol. 63, no. 1, pp. 96-105, Jan. 2014.

[55] D. Simon, Optimal State Estimation: Kalman, $H_{\infty}$, and Nonlinear Approaches. New York, NY, USA: Wiley, 2006.

[56] S. J. Julier and J. K. Uhlmann, "Unscented filtering and nonlinear estimation," Proc. IEEE, vol. 92, no. 3, pp. 401-422, Mar. 2004.

[57] S. Thrun, W. Burgard, and D. Fox, Probabilistic Robotics. Cambridge, MA, USA: MIT Press, 2005.

[58] E. Wan and R. Van der Merwe, "The unscented Kalman filter for nonlinear estimation," in Proc. Adaptive Syst. Signal Process., Commun., Control Symp. IEEE (AS-SPCC), Oct. 2000, pp. 153-158.

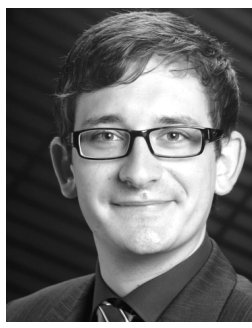

Patrick Schopp received the Dipl.Ing. degree in microsystems engineering from the University of Freiburg, Breisgau, Germany, in 2008, where he is currently pursuing the Ph.D. degree with the Fritz Huettinger Chair of Microelectronics.

His current research interests include state estimation, calibration, and machine learning.

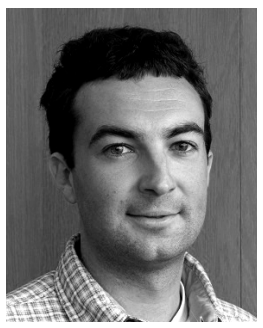

Hagen Graf received the M.Sc. degree in microsystems engineering from the University of Freiburg, Breisgau, Germany, in 2011, where he is currently pursuing the Ph.D. degree with the Fritz Huettinger Chair of Microelectronics.

His current research interests include orientation estimation and application specific processors. 


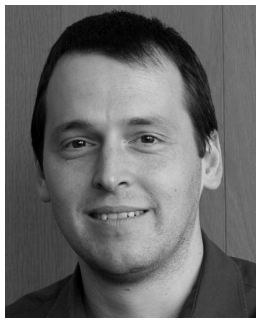

Michael Maurer received the Dipl.Ing. (Hons.) degree in microsystems engineering from the University of Freiburg, Breisgau, Germany, in 2008, where he is currently pursuing the Ph.D. degree.

His current research interests include sensors and systems, control theory, and circuit design.

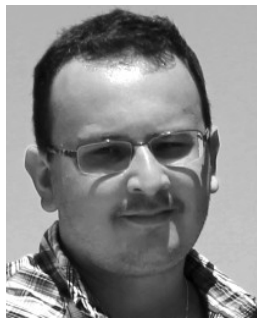

Michailas Romanovas received the B.Sc. degree in electronics engineering from Vilnius Gediminas Technical University, Vilnius, Lithuania, in 2004, and the M.Sc. degree in microsystems engineering from Hochschule Furtwangen University, Furtwangen im Schwarzwald, Germany, in 2006.

He was with NTT Basic Research Laboratories, Atsugi, Japan, from 2006 to 2007. From 2007 to 2014, he was with the Inertial Sensors and Systems Group, Institute for Micro- and Information Technology, Villingen-Schwenningen, Germany. Since 2014, he has been with the German Aerospace Center, Institute of Communications and Navigation, Department for Nautical Systems, Neustrelitz, Germany, where he is involved in multisensor fusion algorithm for maritime applications. His current research interests include inertial sensors, signal processing, localization systems, estimation algorithms, and sensor fusion applications.

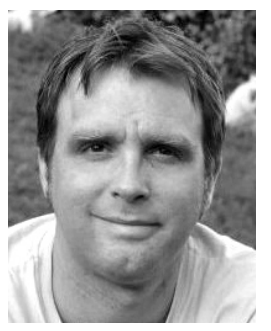

Lasse Klingbeil received the Ph.D. degree in experimental physics from the University of Bonn, Bonn, Germany, in 2006.

Since 2006, he has been conducting research in the wide area of multisensor systems for position and orientation determination of moving objects. From 2006 to 2008, he was a Post-Doctoral Fellow with the Autonomous Systems Laboratory, Commonwealth Scientific and Industrial Research Organization, Brisbane, Australia, where he was involved in sensor networks for agricultural applications and indoor localization. From 2008 to 2011, he was a Research Scientist with the Institute of Microsystems and Information Technology, Hahn-SchickardGesellschaft, Villingen-Schwenningen, Germany, where he was involved in various projects related to inertial sensors, multisensor fusion, and indoor localization. Since 2012, he has been the Head of the GNSS and Moving Objects Group with the Institute of Geodesy and Geoinformation, University of Bonn, where he is involved in mobile mapping with various platforms, GNSS/INS fusion algorithms, and systems and agricultural applications.

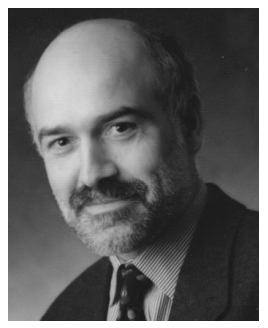

Yiannos Manoli (M'82-SM'08) was born in Famagusta, Cyprus, in 1954. He received the B.A. (summa cum laude) degree in physics and mathematics from Lawrence University, Appleton, WI, USA, in 1978, the M.S. degree in electrical engineering and computer science from the University of California at Berkeley, Berkeley, CA, USA, in 1980, and the Dr. Ing. degree in electrical engineering from Gerhard Mercator University, Duisburg, Germany, in 1987.

He was a Research Assistant with Dortmund University, Dortmund, Germany, from 1980 to 1984, in the field of A/D and D/A converters. In 1985, he joined the newly founded Fraunhofer Institute of Microelectronic Circuits and Systems, Duisburg, where he established a design group involved in mixed-signal CMOS circuits, in particular, for monolithic integrated sensors and application specific microcontrollers. From 1996 to 2001, he held the Chair of Microelectronics as a Full Professor with the Department of Electrical Engineering, University of Saarland, Saarbrücken, Germany. In 2001, he joined the Department of Microsystems Engineering, Institute for Microsystems Technology, Faculty of Engineering, University of Freiburg, Breisgau, Germany, where he established the Chair of Microelectronics. In 2000, he was involved in a research project with Motorola (now Freescale), Phoenix, AZ, USA. In 2006, he spent his sabbatical semester with Intel, Santa Clara, CA, USA, where he was involved in the readout electronics for a high-resolution accelerometer. With an endowment of the Fritz Huettinger Foundation and in memory of the founder of today's Huettinger Elektronik, the University of Freiburg renamed the Chair to Fritz Huettinger Chair of Microelectronics, in 2010. In 2012, he became the Dean of Engineering after having served as an Associate Dean since 2008. Since 2005 , he has additionally served as one of the three directors at the Institute of Micromachining and Information Technology, Hahn-Schickard Gesellschaft, Villingen-Schwenningen, Germany. His current research interests are the design of low-voltage/low-power mixedsignal CMOS circuits, energy harvesting electronics, sensor read-out circuits as well as Analog-toDigital converters. Additional research activities concentrate on motion and vibration energy transducers and on the field of inertial sensors and sensor fusion.

Prof. Manoli has served as a Distinguished Lecturer of the IEEE and as the Guest Editor of the IEEE TRANSACTIONS ON VERY LARge Scale INTEGRATION and the Journal of Solid-State Circuits. He is on the Senior Editorial Board of the IEEE JOURNAL ON EMERGING AND SELECTED TOPICS IN CIRCUITS AND SYSTEMS and on the Editorial Board of the Journal of Low Power Electronics. He has served on the committees of a number of conferences such as the IEEE International Solid-State Circuits Conference, the IEEE European Solid-State Circuits Conference, the IEEE International Electron Devices Meeting, and the IEEE International Conference on Computer Design (ICCD), and was a Program Chair and the Genera Chair of the IEEE ICCD in 2001 and 2002, respectively. He is a member of VDE, Phi Beta Kappa, and Mortar Board. He and his group have received the best paper awards at ESSCIRC 1988, 2009, and 2012, PowerMEMS 2006, MWSCAS 2007, and MSE 2007. The MSE-2007 award was granted for SpicyVOLTsim, a web-based application for the animation and visualization of analog circuits for which he also received the Media Prize of the University of Freiburg in 2005. He was the first to receive the Best Teaching Award of the Faculty of Engineering when it was introduced in 2008. For his creative and effective contributions to the teaching of microelectronics, he has also received the Excellence in Teaching Award of the University of Freiburg and the Teaching Award of the State of Baden-Wuerttemberg, both in 2010. 